\title{
Spin effects in the antler event topology at hadron colliders
}

\author{
Lisa Edelhäuser, ${ }^{a}$ Konstantin T. Matchev ${ }^{b}$ and Myeonghun Park ${ }^{c}$ \\ ${ }^{a}$ Institute for Theoretical Particle Physics and Cosmology, RWTH Aachen University, \\ Aachen, Germany \\ ${ }^{b}$ Physics Department, University of Florida, \\ Gainesville, FL 32611, U.S.A. \\ ${ }^{c}$ TH Division, CERN, \\ Geneva, Switzerland \\ E-mail: ledelhaeuser@physik.rwth-aachen.de, matchev@phys.ufl.edu, \\ Myeonghun.Park@cern.ch
}

ABStRaCT: We investigate spin correlation effects in the "antler" event topology $p p \rightarrow$ $A\left(A^{*}\right) \rightarrow B_{1} B_{2} \rightarrow\left(\ell^{-} C_{1}\right)\left(\ell^{+} C_{2}\right)$ at the LHC. We study the shapes of several kinematic variables, including the relative pseudorapidity, relative azimuthal angle and the energies of the two leptons, as well as several mass variables $M_{\ell \ell}, M_{\mathrm{eff}}, \sqrt{s}_{\mathrm{min}}, M_{T 2}, M_{\mathrm{CT}}$ and $M_{\mathrm{CT} x}$. We focus on the two kinematic extremes of $\sqrt{s}$ - threshold and infinity - and derive analytical expressions for the differential distributions of several variables, most notably the $\cos \theta_{\ell^{-} \ell^{+}}^{*}$ variable proposed by Barr in hep-ph/0511115. For all possible spin assignments of particles $\mathrm{A}, \mathrm{B}$ and $\mathrm{C}$, we derive the $\cos \theta_{\ell^{-} \ell^{+}}^{*}$ differential distribution at threshold, including the effects of spin correlations. Our analytical results help identify the problematic cases for spin discrimination.

Keywords: Supersymmetry Phenomenology, Hadronic Colliders

ArXIV EPRINT: 1205.2054 


\section{Contents}

1 Introduction and motivation $\quad 2$

2 Setup, notation and conventions 5

2.1 Kinematics 5

$\begin{array}{lll}2.2 & \text { Spin assignments } & 7\end{array}$

$\begin{array}{lll}2.3 & \text { Coupling assignments } & 7\end{array}$

$\begin{array}{lll}3 & \text { Kinematic variables for the antler topology } & 10\end{array}$

$\begin{array}{lll}3.1 & \text { The relative pseudorapidity variable } \cos \theta_{\ell^{-} \ell^{+}} & 10\end{array}$

$\begin{array}{ll}3.2 & \text { Azimuthal angular difference } \Delta \varphi\end{array}$

3.3 Invariant mass $M_{\ell^{+} \ell^{-}} \quad 11$

3.4 Contransverse mass $M_{\mathrm{CT}} \quad 11$

3.5 Doubly projected contransverse mass $M_{\mathrm{CT} x} \quad 12$

$\begin{array}{lll}3.6 & \text { Effective mass } M_{\text {eff }} & 12\end{array}$

$\begin{array}{lll}3.7 & \text { The } \sqrt{s}_{\text {min }} \text { variable } & 12\end{array}$

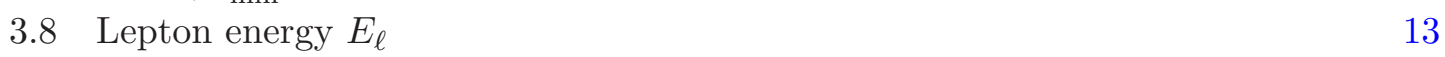

$\begin{array}{ll}3.9 & \text { The Cambridge } M_{T 2} \text { variable } \\ \end{array}$

4 Comparison of different variables $\quad 14$

4.1 Kinematic dependence on $\sqrt{s}$ and the mass spectrum 16

$5 \quad$ Expected shapes in the absence of spin correlations $\quad 18$

$\begin{array}{lll}5.1 & \text { The relative pseudorapidity variable } \cos \theta_{\ell^{-} \ell^{+}} & 19\end{array}$

$\begin{array}{ll}5.2 \text { Azimuthal angular difference } \Delta \varphi & 21\end{array}$

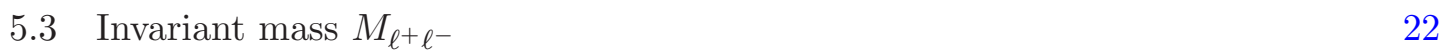

5.4 Doubly projected contransverse mass $M_{\mathrm{CT} x} \quad 22$

5.5 Lepton energy $E_{\ell} \quad 24$

6 Spin correlations in the $\mathcal{C}_{\mathrm{B}}$ distribution $\quad \mathbf{2 4}$

6.1 Spin effects at threshold 24

6.2 Spin effects in the large energy limit 28

$\begin{array}{ll}6.3 & \text { Spin effects near threshold } \\ \end{array}$

7 Comparison of the different spin scenarios $\quad 30$

8 Discussion $\quad 35$

A Derivation of the $\mathcal{C}_{\mathrm{B}}$ angular distribution at threshold $\quad 36$

$\begin{array}{lll}\text { A.1 } & \mathcal{C}_{\mathrm{B}} \text { distribution with no spin correlation } & 36\end{array}$

$\begin{array}{lll}\text { A.2 } & \mathcal{C}_{\mathrm{B}} \text { distribution when particle } B \text { is a fermion } & 36\end{array}$

$\begin{array}{lll}\text { A.3 } & \mathcal{C}_{\mathrm{B}} \text { distribution in the SVF scenario } & 37\end{array}$

$\begin{array}{lll}\text { A.4 } & \mathcal{C}_{\mathrm{B}} \text { distribution in the VVF scenario } & 38\end{array}$

\begin{tabular}{llr} 
A.5 & $\mathcal{C}_{\mathrm{B}}$ distribution in SUSY and MUED & 39 \\
\hline
\end{tabular} 


\section{Introduction and motivation}

Once a signal of new physics is discovered at the Large Hadron Collider (LHC) at CERN, it must be interpreted in terms of the production of new particles with definite properties: mass, spin, charge, coupling strength, chirality, etc. Typically, one studies the properties of new, short-lived resonances by examining their decay products which are seen in the detector. For example, the electric charge of the parent particle (a short-lived resonance) is easily obtained by adding up the electric charges of its daughters (particles from a decaying process), the mass is similarly determined by forming the combined invariant mass of the daughter particles, etc.

Notoriously, this procedure breaks down when some of the daughter particles are very weakly interacting particles and are not visible in the detector (the prototypical examples being the neutrinos of the Standard Model (SM) or hypothetical dark matter WIMPs). Mass and spin measurements then become very challenging, especially at hadron colliders where the partonic center-of-mass (CM) energy and total longitudinal momentum in the event are a priori unknown. This has prompted a long line of research into developing new kinematic methods for determining the masses (see, e.g. [1, 2] and references therein) and spins [3-34] of new semi-invisibly decaying parent particles.

In this paper we tackle the more challenging of those two issues, namely, the determination of the intrinsic spin of the new particles. Broadly speaking, most methods proposed so far in the literature fall into the following categories:

- Methods applicable to the case of lepton colliders, where the CM energy and longitudinal momentum of the initial state are known [4, 17]. Here we shall be interested in hadron colliders like the LHC, where those methods would not be directly applicable.

- Methods attempting either exact [27] or approximate [21] reconstruction of the kinematics of the invisible particles on an event by event basis. Unfortunately, in order to be able to solve for the missing momenta, one needs a relatively long decay chain, with a sufficiently large number of intermediate resonances [35]. In contrast, in this paper we shall consider the case of the shortest decay chain possible (a chain with a single two-body decay), for which there are not enough kinematic constraints for reconstructing the missing momenta, so that one has to resort to likelihood methods [26, 29].

- Methods which study the shapes of the invariant mass distributions of the visible particles originating from one given decay chain $[3,5,6,11,12]$. These methods again require a relatively long decay chain, which would produce at least two visible SM particles, and are not applicable to the short decay chain which will be considered here.

- Methods which simultaneously utilize the measured visible particles from both decay chains. ${ }^{1}$ Here one studies the distributions of suitably defined quantities, e.g. angular

\footnotetext{
${ }^{1}$ In a typical new physics model, the longevity of the dark matter particle is enforced by an exact symmetry, the simplest option being $Z_{2}$ parity, under which all SM particles are even, while the new particles (superpartners, KK-partners, etc.) are odd. A consequence of such $Z_{2}$ parity is that the new particles are pair-produced, and each event has two cascade decays.
} 
variables [8], asymmetry-like variables [29, 33], or global event variables like $M_{\text {eff }}$ or $E_{T}[30]$.

In this paper we shall focus on the most challenging case for spin determination: that of a single stage, two-body decay chain, in which a new parent particle $B$ is produced and then decays to a single visible SM particle $c$ and an invisible daughter particle $C$ :

$$
B \rightarrow c+C \text {. }
$$

Motivated by the dark matter problem, we shall further assume that the new particles $C$ and $B$ are $Z_{2}$-parity odd, so that the LHC produces the parent particles in pairs:

$$
p p \rightarrow B \bar{B} \rightarrow c \bar{c} C \bar{C} .
$$

In general, the process (1.2) can be due to $s, t$ and $u$ channel diagrams. Which of those diagrams are present and/or dominant is a very model-dependent issue, which also depends on the nature of the visible particle $c$. For example, if $c$ carries color (i.e. it is a quark or a gluon), one generically expects all three types of diagrams to be present. On the other hand, if $c$ is a SM lepton, which carries lepton number but no color, then it is reasonable to expect (1.2) to be mediated by $s$-channel diagrams only. ${ }^{2}$ In what follows, for simplicity we shall assume that the SM particle $c$ is a lepton $\ell$, and that the process (1.2) is due to the exchange of a single $Z_{2}$-parity even particle $A$ in an $s$-channel. The resulting event topology is shown in figure 1 and has been dubbed "the antler event topology" [36], due to its resemblance to the headgear of the common wildlife species in Wisconsin. In general, the $s$-channel particle $A$ in figure 1 can be a SM gauge boson (a photon or a $Z$-boson), a SM Higgs boson, or some other new bosonic particle. Depending on the relative masses of particles $A$ and $B$, the $s$-channel resonance $A$ could be on-shell (if $M_{A}>2 M_{B}$ ) or off-shell (if $M_{A}<2 M_{B}$ ). In the following we shall discuss both of those cases.

In summary, as illustrated in figure 1 , the process under study here will be

$$
p p \rightarrow A\left(A^{*}\right) \rightarrow B_{1} B_{2} \rightarrow \ell^{+} \ell^{-} C_{1} C_{2} .
$$

An important benefit of considering the leptonic (i.e. $c=\ell$ ) version of the antler topology is that it does not suffer from large QCD backgrounds (as would be the case if $c$ were a jet). Another advantage is that it allows us to safely focus on the $s$-channel topology only, where we only need to introduce the very minimum number of unknown parameters (see section 2 below) and tackle the problem of spin measurements in a rather model independent way.

In this paper, we shall analyze the kinematics of the antler event topology (1.3) with several goals in mind:

- First, we shall perform a comparative study of the several kinematic variables already discussed in the literature, supplementing them with a few variables of our own (see

\footnotetext{
${ }^{2}$ In principle, $t$ and $u$ channel diagrams cannot be ruled out completely — for example, the dark matter particle $C$ itself may also carry lepton number (although the sneutrino option in supersymmetry is disfavored by experiment), or the $t$ and $u$ channel diagrams could be mediated by exotic particles carrying both color and lepton number (e.g. leptoquarks), or the process (1.2) may involve lepton number violating interactions.
} 


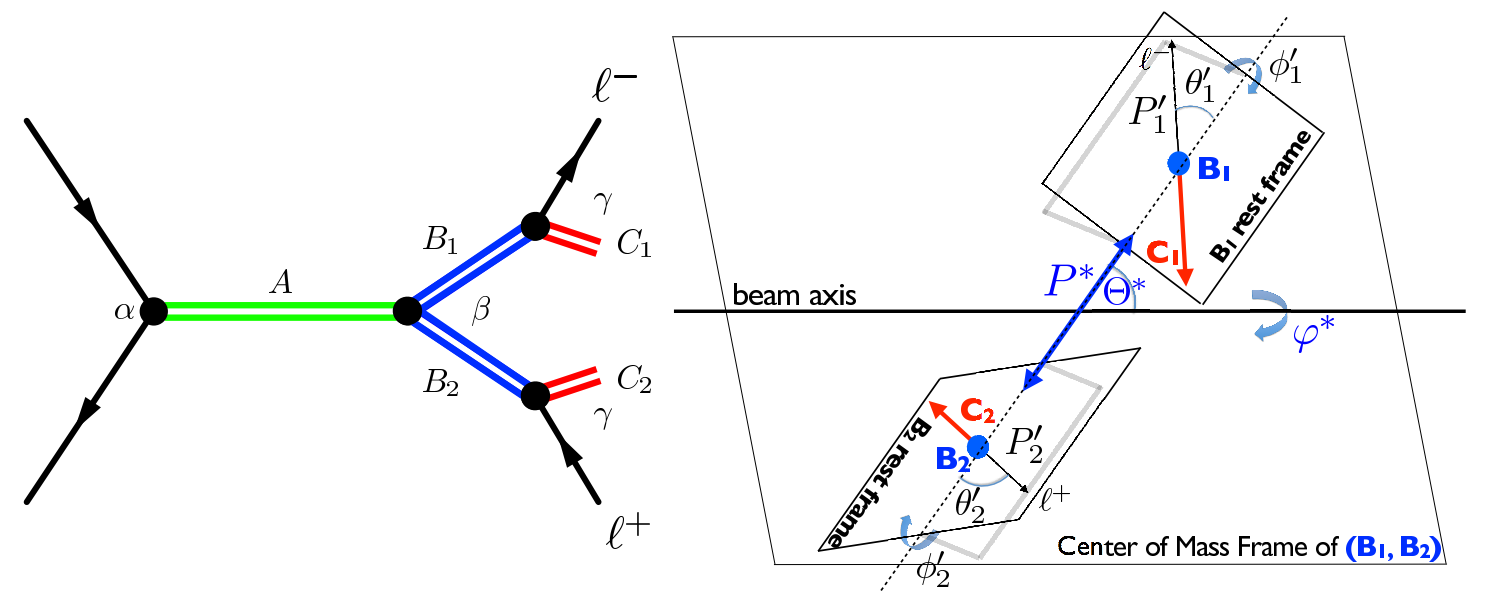

Figure 1. Left: the antler event topology under consideration in this paper. $B_{i}$ and $C_{i}$ are new $Z_{2}$-odd particles, whose spins (as well as the chirality $\beta$ and $\gamma$ of their couplings) are a priori unknown. The intermediate resonance $A$ is a $Z_{2}$-even particle which may or may not be on-shell, and furthermore, its spin (and the chirality $\alpha$ of its coupling to the initial state partons) is also unknown. Right: pictorial definition of the kinematic variables used in this paper. Starred quantities refer to the CM frame of the $B_{1} B_{2}$ system while primed variables refer to the individual rest frames of the parent particles $B_{i}$.

section 3). We shall be mostly interested in the question to what extent the shapes of the different kinematic distributions are sensitive to spin effects. The main objective of this initial study (presented below in section 4) will be to identify the kinematic variables which retain the largest amount of spin information, and are thus most suitable for measuring the spins of particles $A, B$ and $C$.

- Next, we shall address the question how to infer the presence of spin effects in the distribution of any given kinematic variable. Clearly, in order to firmly establish the presence of spin effects, one must first know the expected shape of the distribution in the absence of any spin correlations, i.e. in the pure "phase space approximation". Then, any deviation from the expected "phase space" distribution will indicate spin effects, although a priori it will not be immediately clear whether those are due to non-trivial intrinsic spins of the particles $\{A, B, C\}$, non-trivial coupling chiralities, or some combination of the two [20]. In section 5 we therefore provide the expected "phase space" distributions for several kinematic variables, including the angular variable $\cos \theta_{\ell^{-} \ell^{+}}^{*}$ proposed in [8] (see section 3.1 below for its exact definition). We shall pay special attention to the dependence of the kinematic variables on the partonlevel CM energy $\sqrt{s}$ in the event, and in section 5.1 we shall derive a new formula for the shape of the $\cos \theta_{\ell^{-} \ell^{+}}^{*}$ distribution at threshold $\left(\sqrt{s}=2 M_{B}\right)$.

- Having observed a deviation from the expected "pure phase space" kinematic shape, one can be sure that non-trivial spin effects are in play. But how much of the effect is due to the spins and how much is due to the chirality structure of the couplings? This question is tackled next in section 6. In order to explicitly separate the two effects, 
we derive analytically the $\cos \theta_{\ell^{-} \ell^{+}}^{*}$ distributions at threshold for various spin assignments and arbitrary chiralities. These results provide useful intuition and guidance for the study which comes next - the measurement of the spins of particles $A, B$ and $C$.

- section 7 contrasts the shapes of the $\cos \theta_{\ell^{-} \ell^{+}}^{*}$ distributions obtained for various spin and chirality assignments. The ultimate goal there is to identify which spin combinations for the particles $\{A, B, C\}$ can be distinguished (at least in principle) in such a model-independent way (i.e. without any additional assumptions about the chirality of the fermion couplings) and which can be confused with each other. We shall specify the dangerous "twin" spin scenarios with similar $\cos \theta_{\ell^{-} \ell^{+}}^{*}$ distributions and discuss the possibility to discriminate them through the distributions of other, complementary kinematic variables.

Our study is complementary to other approaches in the literature, which perform a likelihood fit of the full data to a given (mass and spin) hypothesis, using the appropriate matrix element for the given final state [26, 37, 38]. The advantage of such matrix element techniques is that one is using the full information available to the experimenter (taking one-dimensional projections, as we do here, inevitably leads to some information loss). On the other hand, the study of suitable one-dimensional distributions is a very common technique which has important practical benefits: it is simple, does not require a lot of computing power, and does not involve any integrations over unknown momentum components, masses, etc.

Before taking up our four goals, we first introduce our setup and notation in section 2 .

\section{Setup, notation and conventions}

In this paper we study the process (1.3) depicted in figure 1 . The two particles $C_{1}$ and $C_{2}$ are invisible in the detector, while the two massless leptons $\ell^{+}$and $\ell^{-}$are visible. The double lines in the antler event diagram of figure 1 denote particles whose spin is unknown and needs to be determined. The masses of the particles $A, B_{i}$ and $C_{i}$ are also initially unknown, but can eventually be determined from purely kinematic methods [35, 36, 39-49], in spite of the challenge presented by the short decay chain. In what follows, we shall therefore assume that the masses $M_{A}, M_{B}$ and $M_{C}$ of the new particles have already been measured and we concentrate on the issue of spin determination alone.

In order to perform a model independent spin measurement, we will need to separate pure kinematic effects (see section 2.1) from spin and chirality variables (see sections 2.2 and 2.3).

\subsection{Kinematics}

In what follows, we shall find it convenient to describe the kinematics of the process (1.3) in terms of variables which refer to three different reference frames (two of those are illustrated in figure 1): 
1. The $L A B$ frame. This is the frame of the experiment, in which the visible particles are measured. Variables related to the LAB frame will carry no special designations, e.g. the 4 -momenta ${ }^{3}$ of the two visible particles (the leptons) will simply be

$$
\begin{aligned}
& P_{\ell^{-}}^{\mu} \equiv P_{1}^{\mu}=\left(E_{1}, \vec{P}_{1 T}, P_{1 z}\right)=\left(E_{1}, P_{1}, \Theta_{1}, \varphi_{1}\right) ; \\
& P_{\ell^{+}}^{\mu} \equiv P_{2}^{\mu}=\left(E_{2}, \vec{P}_{2 T}, P_{2 z}\right)=\left(E_{2}, P_{2}, \Theta_{2}, \varphi_{2}\right),
\end{aligned}
$$

where $\Theta_{i}$ and $\varphi_{i}$ are the usual spherical angular coordinates. Note that the angles $\Theta_{i}$ are measured from the beam axis (z-axis) and $\varphi_{i}$ are measured in the fixed plane (common to all events) transverse to the beam. Since the leptons are assumed to be massless, we also have $P_{i}=E_{i}$. The LAB variables are not shown in figure 1 .

2. $C M B B$ : the $C M$ frame of the parent pair $B_{1} B_{2}$. This is the relevant frame for describing the $2 \rightarrow 2$ process $p p \rightarrow B_{1} B_{2}$ and the corresponding kinematic variables will be denoted with an asterisk (figure 1). The final state of the $B_{1} B_{2}$ pair is described by three degrees of freedom, which can be taken to be: the CM energy $\sqrt{s^{*}}$ and the spherical angular coordinates of particle $B_{1}$, which are $\Theta^{*}$ (still measured from the beam axis) and $\varphi^{*}$. Since $\sqrt{s^{*}}$ and $\varphi^{*}$ are invariant under longitudinal boosts, they are the same in the LAB frame:

$$
\begin{aligned}
\sqrt{s^{*}} & =\sqrt{s}, \\
\varphi^{*} & =\varphi,
\end{aligned}
$$

so that in what follows for simplicity we shall omit the asterisks on $\sqrt{s}$ and $\varphi$. Instead of $\sqrt{s}$, one could equivalently consider the (magnitude of the) momentum $P^{*}$ of the $B_{i}$ parent particle

$$
P^{*}=\frac{\sqrt{s}}{2}\left(1-\frac{4 M_{B}^{2}}{s}\right)^{\frac{1}{2}},
$$

or the corresponding boost factor $\eta^{*}$

$$
\eta^{*}=\cosh ^{-1}\left(\frac{\sqrt{s}}{2 M_{B}}\right)=\sinh ^{-1}\left(\frac{P^{*}}{M_{B}}\right) .
$$

The main variables $P^{*}, \Theta^{*}$ and $\varphi^{*}$ of the CMBB frame are depicted in figure 1 in blue.

3. $C M B 1$ (CMB2): the $C M$ frame of an individual parent $B_{i}$. The kinematics of each final state lepton is most easily described in the rest frame of its parent particle, where variables will be denoted by a "prime" superscript (figure 1) and will also carry a subscript "1" or "2" to indicate the CM frame of $B_{1}$ CMB1 or the CM frame of $B_{2}$ CMB2. The three degrees of freedom describing the kinematics of each lepton can be taken to be: the magnitude of the momentum $P_{i}^{\prime}$, the polar angle $\theta_{i}^{\prime}$ and the azimuthal angle $\phi_{i}^{\prime}$. Note that, as shown in figure 1 , the polar angles $\theta_{i}^{\prime}$ are measured

\footnotetext{
${ }^{3}$ Here and below, components of 4-momenta are labelled with Greek indices $\mu, \nu, \ldots$.
} 
with respect to the direction of the parent particle $P_{i}$ and not the beam axis, as was the case for the angle $\Theta$. Similarly, the azimuthal angles $\phi_{i}^{\prime}$ are measured differently from the azimuthal angle $\varphi$ in the CMBB frame. The momentum of each lepton in the corresponding parent $\mathrm{CM}$ frame (CMB1 or CMB2) is a constant which only depends on the mass spectrum:

$$
P_{\ell}^{\prime} \equiv P_{1}^{\prime}=P_{2}^{\prime}=\frac{M_{B}}{2}\left(1-\frac{M_{C}^{2}}{M_{B}^{2}}\right) .
$$

In place of the polar angle $\theta_{i}^{\prime}$ one could alternatively use the corresponding pseudorapidity

$$
\eta_{i}^{\prime}=-\ln \left[\tanh \left(\frac{\theta_{i}^{\prime}}{2}\right)\right] .
$$

The frame CMB1 (CMB2) is obtained from the frame CMBB by boosting with a Lorentz factor $\gamma=\cosh \eta^{*}$ along the direction of $B_{1}\left(B_{2}\right)$.

\subsection{Spin assignments}

Since the particles $A, B$ and $C$ are not directly measured, their spins are a priori unknown, and each can be a spin zero scalar $(\mathrm{S})$, a spin $\frac{1}{2}$ fermion $(\mathrm{F})$, a spin 1 vector particle $(\mathrm{V})$, etc. Following [11, 20], we shall consider all allowed spin assignments

$$
(\mathrm{ABC}) \in\{(\mathrm{SSF}),(\mathrm{SVF}),(\mathrm{VSF}),(\mathrm{VVF}),(\mathrm{SFS}),(\mathrm{VFS}),(\mathrm{SFV}),(\mathrm{VFV})\}
$$

in the antler event topology of figure 1 with spins up to one, i.e. consistent with renormalizable interactions. The Feynman diagrams for these 8 cases are listed in table 1, together with a representative example from a popular new physics model with an on-shell particle $A$ (the photon, $Z$-boson and Higgs boson of the SM provide analogous examples with an off-shell $\left.A^{*}\right)$.

For the underlying $2 \rightarrow 2$ process $p p \rightarrow B B$, there are in principle 6 possibilities for the spins of the $(\mathrm{AB})$ pair:

$$
(\mathrm{AB}) \in\{(\mathrm{SS}),(\mathrm{SV}),(\mathrm{VS}),(\mathrm{VV}),(\mathrm{SF}),(\mathrm{VF})\}
$$

which will be discussed in sections 6.2 and 6.3 .

\subsection{Coupling assignments}

As we shall see below, the shapes of the kinematic distributions are affected not just by the spins of the intermediate particles, but also by the chiralities of their couplings. Since the observed particles (leptons) are fermions, the corresponding vertices of the antler diagram may contain chiral projectors. In the spirit of refs. [20,34], in each vertex of the antler diagram, we allow for an arbitrary linear combination of right-handed and left-handed couplings. For example, when $C$ is a fermion and $B$ is a vector boson, the $B \ell C$ coupling is given by

$$
\mathcal{L}_{\text {int }}=\bar{\psi}_{l} \gamma_{\mu}\left(c_{L} P_{L}+c_{R} P_{R}\right) \psi_{C} A_{B}^{\mu},
$$




\begin{tabular}{|c|c|c|}
\hline Spin assignments & Antler diagram & Examples \\
\hline $\mathrm{SSF}$ & & $H_{2}^{0} \rightarrow H_{1}^{+} H_{1}^{-} \rightarrow \ell^{+} \ell^{-} \nu_{1} \bar{\nu}_{1}$ \\
\hline SVF & & $H^{0} \rightarrow W^{+} W^{-} \rightarrow \ell^{+} \ell^{-} \nu \bar{\nu}$ \\
\hline SFS & & $H_{2} \rightarrow \ell_{1}^{+} \ell_{1}^{-} \rightarrow \ell^{+} \ell^{-} B_{H 1} B_{H 1}[50]$ \\
\hline $\mathrm{SFV}$ & & $H_{2} \rightarrow \ell_{1}^{+} \ell_{1}^{-} \rightarrow \ell^{+} \ell^{-} B_{1} B_{1}[50]$ \\
\hline VSF & & $Z^{\prime} \rightarrow \tilde{\ell}^{+} \tilde{\ell}^{-} \rightarrow \ell^{+} \ell^{-} \tilde{\chi}_{1}^{0} \tilde{\chi}_{1}^{0}[36,51]$ \\
\hline VVF & & $Z^{\prime} \rightarrow W^{+} W^{-} \rightarrow \ell^{+} \ell^{-} \nu \bar{\nu}[52]$ \\
\hline VFS & & $Z_{2} \rightarrow \ell_{1}^{+} \ell_{1}^{-} \rightarrow \ell^{+} \ell^{-} B_{H 1} B_{H 1}[53,54]$ \\
\hline VFV & & $Z_{2} \rightarrow \ell_{1}^{+} \ell_{1}^{-} \rightarrow \ell^{+} \ell^{-} B_{1} B_{1}[6,55]$ \\
\hline
\end{tabular}

Table 1. The 8 different spin assignments for the unknown particles $A, B$ and $C$ in the antler topology of figure 1 . The last column lists a few typical examples from popular theoretical models, where supersymmetric particles are denoted by a tilde, while a subscript $n$ indicates a level $n$ Kaluza-Klein mode in theories with Universal Extra Dimensions.

with arbitrary coefficients $c_{L}$ and $c_{R}$. As indicated in figure 1 , the relative chirality of that vertex will then be parameterized in terms of a single parameter

$$
\gamma=\left(\frac{c_{L}^{2}-c_{R}^{2}}{c_{L}^{2}+c_{R}^{2}}\right)
$$

so that $\gamma=0$ corresponds to a vector-like coupling, while $\gamma= \pm 1$ is a purely chiral coupling.

When $B$ is a fermion, one can extend this technology to the other two vertices as well [20] and define the relative chirality of the $A B_{1} B_{2}$ vertex as

$$
\beta=\left(\frac{b_{L}^{2}-b_{R}^{2}}{b_{L}^{2}+b_{R}^{2}}\right)
$$




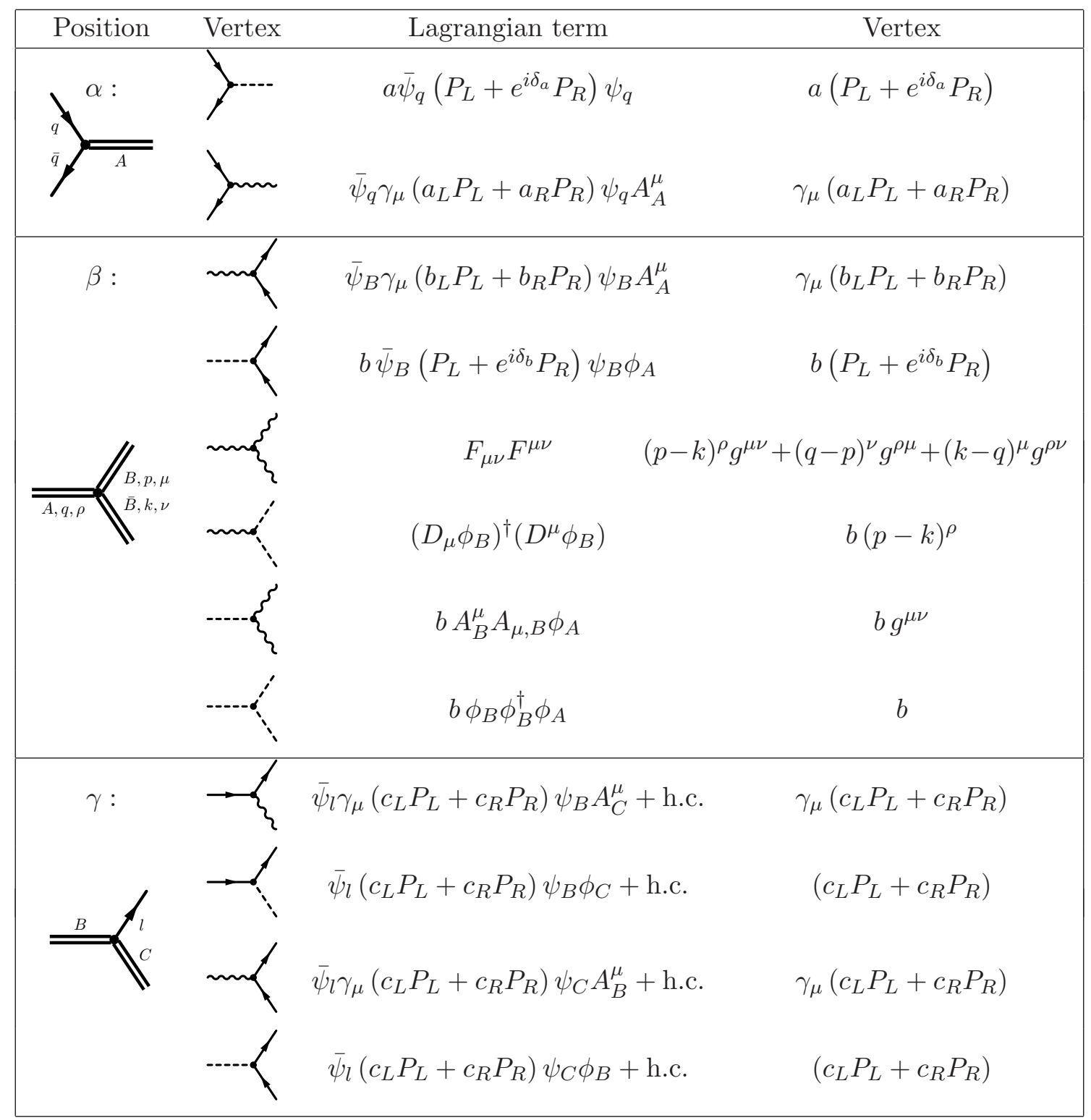

Table 2. Summary of the Lagrangian terms and the corresponding Feynman rules for the vertices in the antler diagram.

and the relative chirality of the $q \bar{q} A$ vertex as

$$
\alpha=\left(\frac{a_{L}^{2}-a_{R}^{2}}{a_{L}^{2}+a_{R}^{2}}\right) .
$$

Our notation is chosen so that the chiral coefficients $a_{L}, a_{R}$ and the relative chirality $\alpha$ refer to the production vertex $q \bar{q} A$ of particle $A ; b_{L}, b_{R}$ and the relative chirality $\beta$ refer to the production vertex $A B_{1} B_{2}$ of particle $B$, while $c_{L}, c_{R}$ and $\gamma$ refer to the production vertex $B \ell C$ of particle $C$.

Table 2 lists the explicit form of the coupling in each vertex for all possible spin assignments for $A, B$ and $C$ which we are considering. 


\begin{tabular}{|c||c|c||c|c||}
\hline \multicolumn{1}{|c||}{ Particle } & \multicolumn{2}{c||}{ SUSY-like } & \multicolumn{2}{c||}{ UED-like } \\
\hline \hline$A$ & $\gamma^{*} / Z^{*}$ & - & $\gamma^{*} / Z^{*}$ & - \\
\hline$B$ & $\tilde{\ell}_{R}$ & 176.62 & $\ell_{1}$ & 505.45 \\
\hline$C$ & $\tilde{\chi}_{1}^{0}$ & 158.18 & $\gamma_{1}$ & 500.89 \\
\hline
\end{tabular}

Table 3. Mass spectrum in GeV for the two study points considered in the text. The SUSY-like mass spectrum corresponds to the CMS LM6 study point [56], while the UED-like spectrum comes from the Minimal UED model with $R^{-1}=500 \mathrm{GeV}, \lambda R=20$ and $m_{h}=120 \mathrm{GeV}$ [4-6].

\section{Kinematic variables for the antler topology}

At a hadron collider like the LHC, the energy and momentum of the initial state partons vary from one event to another. The lack of knowledge about the kinematics of the initial state prevents us from computing the momenta of the missing particles $C_{i}$ in the simple antler topology. (At a linear collider, where the energy and momentum of the initial state are known, the missing momenta in the antler topology can be reconstructed only up to a two-fold ambiguity [17], even assuming precise knowledge of the masses of the intermediate particles). Thus we are limited to variables constructed out of the measured 4-momenta $P_{i}^{\mu}$ (defined in eqs. (2.1)-(2.2)) of the visible particles $\ell^{ \pm}$in the LAB frame. In this section, we review a number of such variables which have been discussed in the literature. In order to gauge their sensitivity to spin effects in the antler topology, we shall investigate their distributions for two conventional study points, whose mass spectra are listed in table 3 . (Notice that both of these models are examples with an off-shell $A^{*}$, since $M_{A}<2 M_{B}$.) For each of these two mass spectra, we shall consider two spin configurations: VSF for SUSY and VFV for UED, postponing the comprehensive analysis including the remaining spin combinations until sections 6 and 7 . The resulting four cases will be contrasted in figure 2, where we shall plot the unit normalized distributions for all variables introduced in this section.

\subsection{The relative pseudorapidity variable $\cos \theta_{\ell^{-} \ell^{+}}^{*}$}

In [8] A. Barr introduced an angular variable $\theta_{\ell^{-} \ell^{+}}^{*}$ which is related to the relative pseudorapidity of the visible particles in the LAB frame as

$$
\begin{aligned}
\mathcal{C}_{\mathrm{B}} \equiv \cos \theta_{\ell^{-} \ell^{+}}^{*} & =\tanh \left(\frac{\Delta \eta_{\ell^{-} \ell^{+}}}{2}\right), \\
\Delta \eta_{\ell^{-} \ell^{+}} & \equiv \eta_{\ell^{-}}-\eta_{\ell^{+}}=\eta_{1}-\eta_{2} .
\end{aligned}
$$

For brevity, from now on we shall use $\mathcal{C}_{\mathrm{B}}$ to denote $\cos \theta_{\ell^{-} \ell^{+}}^{*}$. The pseudorapidities of the two visible particles in the LAB frame are defined as

$$
\eta_{i} \equiv \frac{1}{2} \ln \left(\frac{P_{i}+P_{i z}}{P_{i}-P_{i z}}\right)
$$

in terms of the measured momenta $P_{i}$ and $P_{i z}$ from eqs. (2.1)-(2.2). 


\subsection{Azimuthal angular difference $\Delta \varphi$}

The azimuthal angular difference of the visible particles

$$
\Delta \varphi \equiv \cos ^{-1}\left(\frac{\vec{P}_{1 T} \cdot \vec{P}_{2 T}}{P_{1 T} P_{2 T}}\right)
$$

has also been shown to be sensitive to spin effects $[28,29]$ and we shall include it among our set of observables here as well.

\subsection{Invariant mass $M_{\ell^{+} \ell^{-}}$}

One can also consider the invariant mass of the two visible particles in the antler topology

$$
M_{\ell^{+} \ell^{-}} \equiv \sqrt{\left(P_{1}+P_{2}\right)^{\mu}\left(P_{1}+P_{2}\right)_{\mu}} .
$$

The shape of the $M_{\ell^{+} \ell^{-}}$distribution was studied in detail in [36] for the case of resonant production $\left(M_{A}>2 M_{B}\right)$. It was shown that the $M_{\ell^{+} \ell^{-}}$distribution exhibits a point (called a "cusp"), where it is not continuously differentiable. If the mass spectrum is such that

$$
M_{A} \leq\left(2 M_{B}\right) \cosh \left(\frac{1}{2}\right) \approx(2.26) M_{B}
$$

then the cusp appears at the very peak of the distribution. On the other hand, when $M_{A}>2.26 M_{B}$, the peak is smooth and the cusp appears somewhere below the peak [36].

In what follows, we shall prefer to work with dimensionless variables, so we need to rescale our mass variables by a suitable mass constant. Since the mass spectrum of the antler topology is assumed to be already known, the momentum $P_{\ell}^{\prime}$ of each lepton in its mother's rest frame (2.7) is also known and we can use it for normalization. We therefore define the rescaled invariant mass ${ }^{4}$ as

$$
\hat{M}_{\ell^{+} \ell^{-}}=\frac{M_{\ell^{+} \ell^{-}}}{2 P_{\ell}^{\prime}}
$$

\subsection{Contransverse mass $M_{\mathrm{CT}}$}

The contransverse mass variable

$$
M_{\mathrm{CT}}=\sqrt{2\left(P_{1 T} P_{2 T}+\vec{P}_{1 T} \cdot \vec{P}_{2 T}\right)}
$$

proposed in [57] is also suitable for the antler topology. It is invariant under longitudinal boosts from the LAB frame to the CMBB frame, and under "back to back" boosts of the parent particles. The $M_{\mathrm{CT}}$ distribution has an upper kinematic endpoint

$$
M_{\mathrm{CT}}^{(\max )}=2 P_{\ell}^{\prime}=M_{B}\left(1-\frac{M_{C}^{2}}{M_{B}^{2}}\right)
$$

\footnotetext{
${ }^{4}$ Rescaled quantities will be denoted with a hat.
} 
which can be used for mass measurements [57]. Here we shall focus on the shape of the distribution of the corresponding rescaled $M_{\mathrm{CT}}$ variable

$$
\hat{M}_{\mathrm{CT}}=\frac{M_{\mathrm{CT}}}{2 P_{\ell}^{\prime}} .
$$

eqs. (3.9) and (3.10) imply that the endpoint of the $\hat{M}_{\mathrm{CT}}$ distribution is always at $\hat{M}_{\mathrm{CT}}=1$ and this will be evident in the plots below.

\subsection{Doubly projected contransverse mass $M_{\mathrm{CT} x}$}

The doubly projected contransverse mass variable $M_{\mathrm{CT}_{\perp}}$ was proposed in order to remove the upstream boost effect from initial state radiation (ISR) [43]. $M_{\mathrm{CT}_{\perp}}$ is the onedimensional analogue of (3.8) which uses the transverse momenta components which are orthogonal (hence the subscript " $\perp$ ") to the upstream $\vec{P}_{T}$ which can be caused by ISR or previous decays. Here we shall not consider ISR effects, so in the absence of any upstream $\vec{P}_{T}$, one is free to choose any transverse axis to define the " $\perp$ " components. For concreteness, here we shall choose the $x$-axis and define $M_{\mathrm{CT}_{\perp}}$ as

$$
M_{\mathrm{CT} x}=\sqrt{2\left(\left|P_{1 x} P_{2 x}\right|+P_{1 x} P_{2 x}\right)} .
$$

The corresponding rescaled variable is

$$
\hat{M}_{\mathrm{CT} x}=\frac{M_{\mathrm{CT} x}}{2 P_{\ell}^{\prime}}
$$

and its endpoint is also at $\hat{M}_{\mathrm{CT} x}=1$. For our purposes, $\hat{M}_{\mathrm{CT} x}$ has an important advantage - the analytical formula for its differential distribution (in the absence of spin correlations) is already known [43], providing an important benchmark for spin studies (see section 5.4 below).

\subsection{Effective mass $M_{\text {eff }}$}

The effective mass variable $M_{\text {eff }}$ [58] has been widely utilized in SUSY searches. When applied to the antler topology, it reads

$$
M_{\mathrm{eff}}=P_{1 T}+P_{2 T}+E_{T} .
$$

Here we use the rescaled variable

$$
\hat{M}_{\text {eff }}=\frac{M_{\text {eff }}}{4 P_{\ell}^{\prime}} .
$$

Many SUSY analyses use $M_{\text {eff }}$ as one of their selection cuts, since it is correlated with the mass scale of the particles produced in the event.

\subsection{The $\sqrt{s}_{\text {min }}$ variable}

The $\sqrt{s}_{\min }$ variable was advertized in $[59,60]$ as a better estimator of the mass scale of the hard scattering, since it explicitly accounts for the masses of the invisible particles, and is defined in a theoretically rigorous way. $\sqrt{s}_{\text {min }}$ is the minimum value of $\sqrt{s}$ which is 
required in order to account for the observed visible particles and the measured $\mathbb{E}_{T}$ in the event. In the case of the antler event topology, the general formula for $\sqrt{s}_{\text {min }}$ from [59] reduces to

$$
\sqrt{s}_{\text {min }}=\sqrt{M_{\ell^{+} \ell^{-}}^{2}+\left|\vec{P}_{1 T}+\vec{P}_{2 T}\right|^{2}}+\sqrt{4 M_{C}^{2}+E_{T}^{2}}
$$

already accounting for the fact that the combined mass of all invisible particles present in the event (in this case the two $C^{\prime}$ 's) is $2 M_{C}$. It was noted in [59] that for typical SUSY events the $\sqrt{s}_{\text {min }}$ distribution peaks near the mass threshold $\left(2 M_{B}\right)$ for production of the two parents. This is why we choose to rescale the $\sqrt{s}_{\text {min }}$ variable by $2 M_{B}$ and define

$$
\sqrt{\hat{s}}_{\min }=\frac{\sqrt{s}_{\min }}{2 M_{B}}
$$

which we expect to peak near $\sqrt{\hat{s}}_{\text {min }}=1$, as confirmed in figure 2 .

We anticipate that the $\sqrt{s}_{\text {min }}$ variable will be useful for spin studies, particularly in the off-shell scenario when $M_{A}<2 M_{B}$. In that case, the two $B$ particles are produced near threshold, and the threshold suppression of the $\sqrt{s}$ distribution will be sensitive to the spins of the $A$ and $B$ particles (see section 6.3 below). Since $\sqrt{s}_{\text {min }}$ is designed to correlate with the actual $\sqrt{s}$ in the event, any spin effects present in the $\sqrt{s}$ distribution will be inherited to some extent by the $\sqrt{s}_{\text {min }}$ distribution as well.

\subsection{Lepton energy $E_{\ell}$}

The lepton energy $E_{i}$ is a classic variable for kinematic studies at a linear collider. Here we revisit this variable for the case of a hadron collider, rescaling as usual

$$
\hat{E}_{i}=\frac{E_{i}}{P_{\ell}^{\prime}}
$$

In what follows we shall not distinguish the two lepton energies $E_{1}$ and $E_{2}$ and will always show them together on the same plot as $E_{\ell}$.

\subsection{The Cambridge $M_{T 2}$ variable}

The Cambridge $M_{T 2}$ variable was originally proposed [61] for precisely the antler topology case of figure 1. Since then, it has been extensively applied for measuring the mass spectrum of the new particles $A, B$ and $C$. In the case of the antler topology, $M_{T 2}$ is given by $[44,62]$

$$
\begin{aligned}
M_{T 2}\left(M_{C}\right) & =\sqrt{A_{T}}+\sqrt{A_{T}+M_{C}^{2}}, \\
A_{T} & =\frac{1}{2}\left(P_{1 T} P_{2 T}+\vec{P}_{1 T} \cdot \vec{P}_{2 T}\right) .
\end{aligned}
$$

Just like $\sqrt{s}_{\text {min }}, M_{T 2}$ requires an input mass for the missing particle, which in eq. (3.18) has been taken to be the correct value $M_{C}$. In that case, the $M_{T 2}$ endpoint is equal to the parent mass $M_{B}$ :

$$
M_{T 2}^{(\max )}\left(M_{C}\right) \equiv \max \left\{M_{T 2}\left(M_{C}\right)\right\}=M_{B} .
$$

This motivates us to rescale the $M_{T 2}$ variable as

$$
\hat{M}_{T 2}=\frac{M_{T 2}\left(M_{C}\right)-M_{C}}{M_{B}-M_{C}},
$$


so that the distribution of $\hat{M}_{T 2}$ ranges from 0 to 1 .

In conclusion of this section, we emphasize that this is by far not an exhaustive list there are other variables that could also be usefully applied to the antler event topology. We have limited ourselves here to some of the more popular and established variables. Other recent suggestions include the variables $M_{\mathrm{CT} 2}[47,63], \cos \theta^{V}[33], \mathcal{E}_{T}^{\mathrm{eff}}[64]$, and the boost-invariant observables of [65].

\section{Comparison of different variables}

Having defined the nine kinematic variables of interest in the previous section, we shall now compare their sensitivity to spin effects in the antler topology. In order to get some preliminary idea about this, in figure 2 we show the distributions of our nine variables in the four cases, obtained by pairing the two mass spectra from table 3 (SUSY-like or UED-like) with the corresponding spin assignments (VSF for the MSSM and VFV for MUED). If a particular distribution is affected by spins, one would expect a visible difference between the two dotted lines, showing the two different spin scenarios for the same SUSY-like mass spectrum. Similarly, one would also expect a difference between the two solid lines, which show the two different spin scenarios for a UED-like mass spectrum.

Figure 2 reveals that the nine variables from section 3 exhibit varying degrees of sensitivity to spins. The distributions of some variables, like $M_{\mathrm{CT}}, M_{\mathrm{CT} x}$ and $M_{T 2}$, show very little variation and appear to be relatively insensitive to changes in the spins and masses. The case of $M_{\mathrm{CT} x}$ is particularly striking - all four $M_{\mathrm{CT} x}$ distributions are virtually identical. This begs the question whether the $M_{\mathrm{CT} x}$ distribution has any dependence on the spins at all. This issue will be tackled in section 5.4 below.

At the same time, figure 2 also demonstrates that the remaining 6 variables, $\mathcal{C}_{\mathrm{B}}, \Delta \varphi$, $M_{\ell^{+} \ell^{-}}, M_{\mathrm{eff}}, \sqrt{s}_{\text {min }}$ and $E_{\ell}$, all have a certain degree of sensitivity to spins, and are generally promising for spin studies. However, at this point one should not read too much into figure 2, since the observed variations in the kinematic shapes can be attributed to several different factors, not all of which are related to spins:

- First, we see that in figure 2 there is a noticeable difference between lines of the same color, i.e. when the same spin scenario is shown for two different mass spectra. This means that the shapes of the kinematic distributions are affected by the mass spectrum (which in turn determines the available phase space). This is not necessarily a problem for spin studies per se, since the mass spectrum will be known in advance. However, this does present a problem in the sense that any conclusions that one might draw as to which variables are most sensitive to spins, will necessarily be subject to the choice of mass spectrum. In other words, when it comes to spin determinations, it may very well be that for one particular mass spectrum a certain kinematic variable performs best, but for a different spectrum another variable is the winner. This is why in what follows we shall take great care in illustrating our results throughout the full mass parameter space, and not just a couple of study points as in figure 2 (see section 4.1 below). 

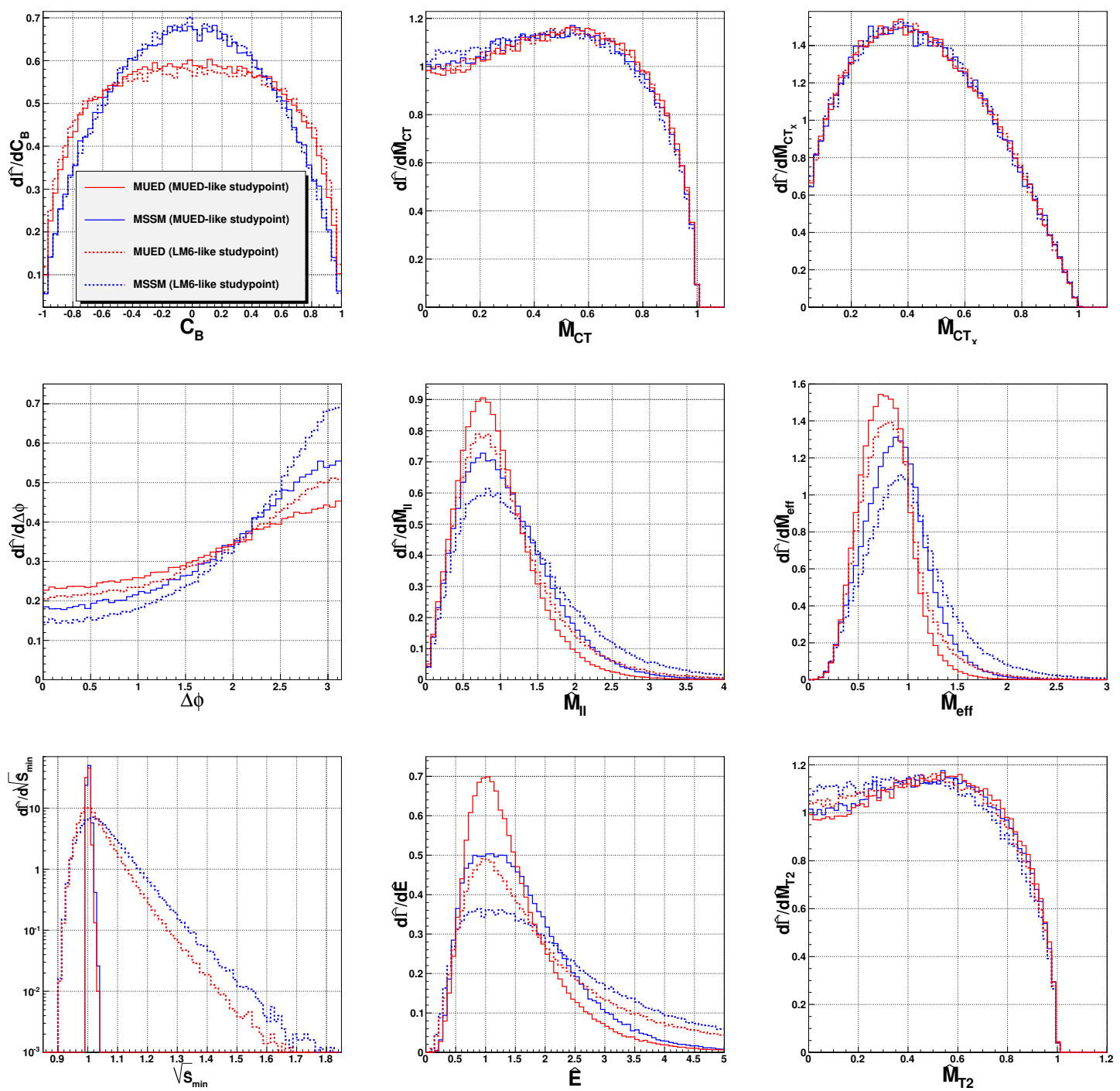

Figure 2. Unit-normalized distributions of the nine variables introduced in section 3 at a $7 \mathrm{TeV}$ LHC. Each panel shows 4 cases, depending on the mass spectrum from table 3: SUSY-like (dotted lines) or UED-like (solid lines); and the spin configuration: VSF for the MSSM (blue lines) and VFV for MUED (red lines).

- One should also keep in mind that the $\sqrt{s}$ distribution also varies from one model point to another, and this will impact the distributions of kinematic variables which carry a dependence on the underlying $\sqrt{s}$ in the event. Figure 3 shows the $\sqrt{s}$ distributions for the four cases discussed in figure 2. As expected, the SUSY spin scenario (VSF) leads to a significantly harder $\sqrt{s}$ distribution (blue lines in figure 3 ), due to the $p$-wave threshold suppression for scalar $B_{1} B_{2}$ production. Now returning to figure 2 , it should be rather intuitive that $\mathcal{C}_{\mathrm{B}}, \Delta \varphi, M_{\ell^{+} \ell^{-}}, M_{\mathrm{eff}}, \sqrt{s}_{\min }$ and $E_{\ell}$ are all sensitive to the value of $\sqrt{s}$, so it is not immediately clear how much of the 




Figure 3. Unit-normalized $\sqrt{s}$ distributions for the four cases presented in figure 2 .

differences between the red and blue lines in their distributions were simply due to the different $\sqrt{s}$ distributions in figure 3 as opposed to spins. Conversely, the variables $M_{\mathrm{CT}}$ and $M_{\mathrm{CT} x}$ are less sensitive to $\sqrt{s}$ because of the back-to-back boost invariance. Therefore they will not be subject to the $\sqrt{s}$ effects in figure 3 , and will have much smaller variations, as indeed observed in figure 2 .

- Of course, the shapes of the kinematic distributions do carry information about spins. As already explained in section 2.2, this information can be extracted by comparing the predictions from the eight different spin scenarios in table 1 to the observed data.

- As emphasized in [20], spin correlations appear not only because of spins, but also due to the chirality of the fermion couplings. In reality, one is measuring the combined effects from spins and chirality, and the blueprint for disentangling these two effects can be found in $[20,23,24,29]$. We shall investigate the chirality effect in section 6 .

\subsection{Kinematic dependence on $\sqrt{s}$ and the mass spectrum}

We shall now discuss in some more detail to what extent our conclusions from the simple exercise in figure 2 persist as we vary the mass spectrum and the energy $\sqrt{s}$ in the event. In figure 4 we compare the VSF spin scenario of the MSSM to the VFV spin scenario in MUED, as a function of $\sqrt{s}$ (plotted on the $x$-axis) and the mass splitting between $B$ and $C$, parameterized through the mass ratio $M_{C} / M_{B}$ (plotted on the $y$-axis), for a fixed $M_{B}=500 \mathrm{GeV}$. Each panel shows a temperature plot of the $\chi^{2}$ measure for one of the nine kinematic variables from figure 2. Larger values of $\chi^{2}$ (warmer colors in figure 4) indicate larger differences in the predicted shapes between the SUSY and MUED scenarios, and smaller values of $\chi^{2}$ indicate rather similar shapes, where the two scenarios might look the same. 

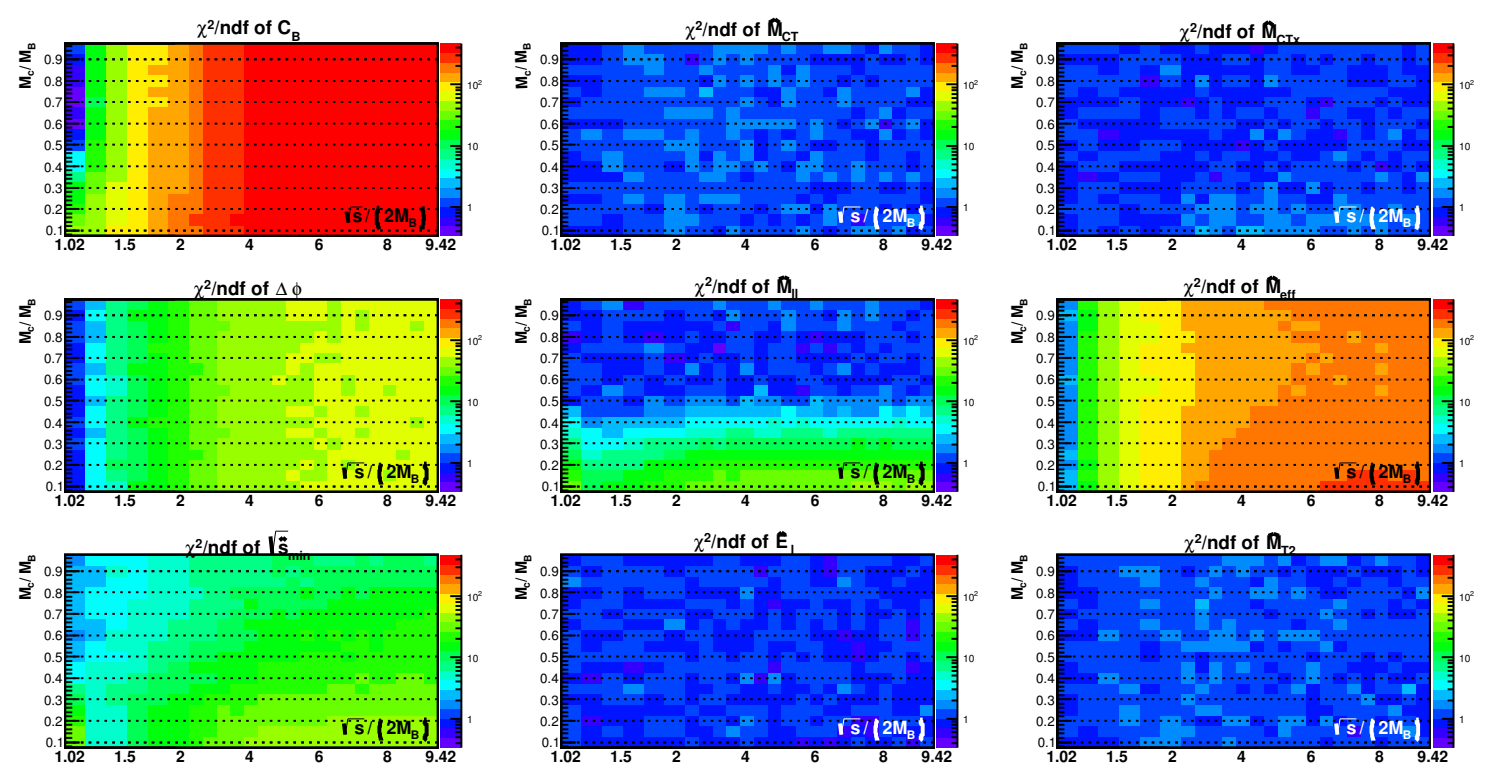

Figure 4. $\chi^{2}$-comparisons of the two spin scenarios (VSF in the MSSM versus VFV in MUED), for each of the nine observables from figure 2. We fix the mass of $B$ as $M_{B}=500 \mathrm{GeV}$, and then vary the CM energy $\sqrt{s}$ and the mass $M_{C}$ of $C$.

As already mentioned, the observed differences in shapes in general could be due to spin correlations, but could also be explained by different kinematics, as suggested by figure 3 . In order to fairly assess the performance of the different kinematic variables with respect to pure spin effects, one should compare the $\chi^{2}$ values on the different panels in figure 4 for a common value of $\sqrt{s}$, thus taking kinematics out of the equation. Then, the variable with the larger $\chi^{2}$ for a given value of $\sqrt{s}$ would be more sensitive to spin correlations, and vice versa.

There are several lessons to be learned from figure 4. First, not surprisingly, different variables exhibit different degrees of sensitivity to spin correlations. In this sense, $M_{\mathrm{CT}}$, $M_{\mathrm{CT} x}, M_{T 2}$ and $E_{\ell}$ appear to be the worst performers. For the first three variables, this might have been expected, based on the result from figure 2. The surprising member of this group is the lepton energy $E_{\ell}$ : its distributions now appear identical in the two spin scenarios, in spite of the much more promising differences seen previously in figure 2 . This suggests that the differences in the $E_{\ell}$ distributions in figure 2 can be attributed solely to the different $\sqrt{s}$ kinematics seen in figure 3 and not to true spin correlations.

Figure 4 also reveals that the contrast between the two spin scenarios depends quite significantly on the energy $\sqrt{s}$, especially in the case of $\mathcal{C}_{\mathrm{B}}, \Delta \varphi, M_{\text {eff }}$ and $\sqrt{s}_{\text {min }}$. In general, as the value of $\sqrt{s}$ increases, differences become more pronounced. For the largest values of $\sqrt{s}$ seen on the plots (several times above threshold) the most discriminating variable appears to be $\mathcal{C}_{\mathrm{B}}$, however such conclusion would be rather premature. As figure 3 shows, at hadron colliders particles are produced near threshold, with typical values of $\sqrt{s}$ only 10-20\% above the $2 M_{B}$ threshold. Unfortunately, figure 4 shows that for those low values of $\sqrt{s}$, the discriminating power of the $\mathcal{C}_{\mathrm{B}}$ variable is significantly reduced, and it behaves similarly to the other alternatives like $M_{\mathrm{eff}}$ and $\Delta \varphi$. 



Figure 5. The same as figure 4 , but instead of a linear collider at a fixed CM energy $\sqrt{s}$, results are shown for the $8 \mathrm{TeV}$ LHC. Here we vary both $M_{B}$ and $M_{C}$ and plot in the $\left(M_{B}, \frac{M_{C}}{M_{B}}\right)$ plane.

In order to get a sense of the full picture at the LHC (currently running at $8 \mathrm{TeV}$ $\mathrm{CM}$ energy), one needs to re-weight the results from figure 4 by the proper $\sqrt{s}$ probability distributions analogous to figure 3 . The result is shown in figure 5 , where this time we vary both $M_{B}$ and $M_{C}$ and plot in the $\left(M_{B}, \frac{M_{C}}{M_{B}}\right)$ plane. Interestingly, figure 5 shows that in practice the variables whose distributions are most likely to show a noticeable difference between SUSY and MUED are $M_{\text {eff }}, \sqrt{s}_{\text {min }}$ and $M_{\ell^{+} \ell^{-}}$. All three of these variables are sensitive to the $\sqrt{s}$ in the event, and are therefore capable of discriminating the different shapes of the $\sqrt{s}$ distributions in figure 3 .

\section{Expected shapes in the absence of spin correlations}

In this section, we shall be interested in analytical predictions of the shapes of some of the observables from the previous two sections. Consider a generic kinematic variable $V$. In general, it is a function of the underlying event kinematics described in section 2.1:

$$
\begin{aligned}
V & =V\left(\sqrt{s}, \Theta^{*}, \varphi ; \theta_{1}^{\prime}, \phi_{1}^{\prime} ; \theta_{2}^{\prime}, \phi_{2}^{\prime}\right), \\
& =V\left(\eta^{*}, \Theta^{*}, \varphi ; \eta_{1}^{\prime}, \phi_{1}^{\prime} ; \eta_{2}^{\prime}, \phi_{2}^{\prime}\right) .
\end{aligned}
$$

Once this function is known, the pure "phase space" distribution of the variable $V$ for a given fixed $\sqrt{s}$ can be obtained by integrating over $d \Omega_{1}^{\prime}$ for the $B_{1} \rightarrow \ell^{-} C_{1}$ decay in the CMB1 frame, over $d \Omega_{2}^{\prime}$ for the $B_{2} \rightarrow \ell^{+} C_{2}$ decay in the CMB2 frame, and over $d \Omega$ for the initial $p p \rightarrow B_{1} B_{2}$ scattering in the CMBB frame:

$$
\left(\frac{\mathrm{d} N}{\mathrm{~d} V}\right)(V, \sqrt{s}) \propto \int d \Omega \int d \Omega_{1}^{\prime} \int d \Omega_{2}^{\prime} \delta\left(V-V\left(\sqrt{s}, \Theta^{*}, \varphi ; \theta_{1}^{\prime}, \phi_{1}^{\prime} ; \theta_{2}^{\prime}, \phi_{2}^{\prime}\right)\right) .
$$


Finally, at a hadron collider one also needs to convolute the fixed $\sqrt{s}$ distribution (5.3) with the parton luminosity $L(\sqrt{s})$ (recall figure 3 ), so that the experimentally observed distribution will be given by

$$
\begin{aligned}
\frac{\mathrm{d} N}{\mathrm{~d} V} & \propto \int d \sqrt{s} L(\sqrt{s})\left(\frac{\mathrm{d} N}{\mathrm{~d} V}\right)(V, \sqrt{s}) \\
& =\int d \sqrt{s} L(\sqrt{s}) \int d \Omega \int d \Omega_{1}^{\prime} \int d \Omega_{2}^{\prime} \delta\left(V-V\left(\sqrt{s}, \Theta^{*}, \varphi ; \theta_{1}^{\prime}, \phi_{1}^{\prime} ; \theta_{2}^{\prime}, \phi_{2}^{\prime}\right)\right) .
\end{aligned}
$$

Unfortunately, even if we concentrate on the fixed $\sqrt{s}$ case of (5.3), the 6 integrations in it are typically quite involved, and exact analytical expressions for the resulting distribution $d N / d V(V, \sqrt{s})$ are known only in very few special cases (reviewed below). To make matters worse, the fixed $\sqrt{s}$ distribution of (5.3) still needs to be integrated numerically over the parton luminosities as in (5.4). Given all those difficulties, analytical studies of the kinematic shapes (5.5) (or even the simpler case of (5.3)) appear to be quite challenging, especially for arbitrary values of $\sqrt{s}$.

In this and the following section, therefore, we shall limit ourselves to a much more manageable task: instead of dealing with a general $\sqrt{s}$, we shall derive analytical formulas in some interesting and relevant $\sqrt{s}$ limits. There are two special values of $\sqrt{s}$, namely the endpoints of its definition interval: the threshold value $\sqrt{s}_{t h}=2 M_{B}$ and the infinite energy limit $\sqrt{s} \rightarrow \infty$. Taking either one of those limits leads to simplifications in the defining functions (5.1), (5.2), and the integrations become easier, although success is not always guaranteed. We shall now discuss selected kinematic observables for general $\sqrt{s}$ (where possible), and in the two limits of $\sqrt{s} \rightarrow \sqrt{s}_{t h}$ and $\sqrt{s} \rightarrow \infty$. In the off-shell case of $M_{A}<2 M_{B}$, the threshold limit is particularly relevant: we have already learned from figure 3 that at the LHC the mother particles $B$ are much more likely to be produced near threshold and not with very large boosts.

\subsection{The relative pseudorapidity variable $\cos \theta_{\ell^{-} \ell^{+}}^{*}$}

We begin with the $\mathcal{C}_{\mathrm{B}}$ variable from section 3.1

$$
\mathcal{C}_{\mathrm{B}}=\tanh \left(\frac{\eta_{1}-\eta_{2}}{2}\right)
$$

The lepton pseudorapidities in the LAB frame $\eta_{1}$ and $\eta_{2}$ (which were defined in (3.3)) can be conveniently expressed in terms of the respective pseudorapidities (2.8) in the CMB1 and CMB2 frames as follows

$$
\eta_{i}=\eta_{z}+\frac{1}{2} \ln \left[\frac{\cosh \left(\eta_{i}^{\prime}-(-1)^{i} \eta^{*}\right)+\cos \Theta^{*} \sinh \left(\eta_{i}^{\prime}-(-1)^{i} \eta^{*}\right)+\cos \phi_{i}^{\prime} \sin \Theta^{*}}{\cosh \left(\eta_{i}^{\prime}-(-1)^{i} \eta^{*}\right)-\cos \Theta^{*} \sinh \left(\eta_{i}^{\prime}-(-1)^{i} \eta^{*}\right)-\cos \phi_{i}^{\prime} \sin \Theta^{*}}\right],
$$

where $\eta_{z}$ is the rapidity of the CM frame. Substituting (5.6) and (5.7) into (5.3), one can in principle obtain the $\mathcal{C}_{\mathrm{B}}$ distribution for a given fixed $\sqrt{s}$. Unfortunately, we have not been able to obtain closed form expressions for general $\sqrt{s}$, and shall consider the two limiting cases instead. 

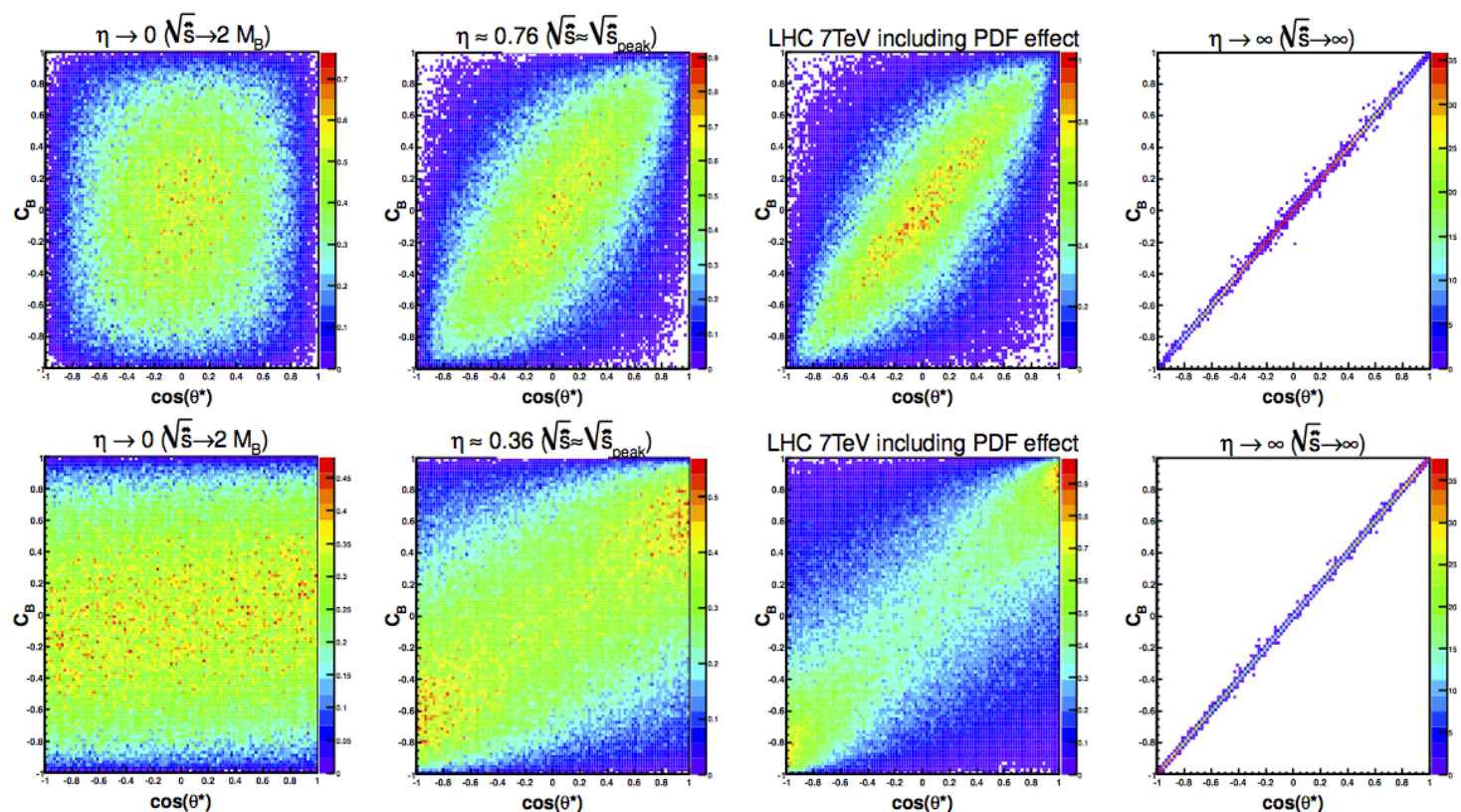

Figure 6. Correlation between $\mathcal{C}_{\mathrm{B}}$ and $\cos \Theta^{*}$, for different boost factors $\eta^{*}$. The upper (lower) row of plots is for the MSSM (MUED) study point from table 3.

In the infinite energy limit $\sqrt{s} \rightarrow \infty$, eq. (2.6) implies that $\eta^{*} \rightarrow \infty$ and then (5.7) reduces to

$$
\begin{aligned}
& \eta_{1} \underset{\eta^{*} \rightarrow \infty}{\longrightarrow} \eta_{z}+\ln \left[\cot \left(\frac{\Theta^{*}}{2}\right)\right], \\
& \eta_{2} \underset{\eta^{*} \rightarrow \infty}{\longrightarrow} \eta_{z}+\ln \left[\tan \left(\frac{\Theta^{*}}{2}\right)\right] .
\end{aligned}
$$

Then (5.6) simplifies to

$$
\mathcal{C}_{\mathrm{B}} \underset{\eta^{*} \rightarrow \infty}{\longrightarrow} \cos \Theta^{*}
$$

Therefore, in the infinite energy limit, the $\mathcal{C}_{\mathrm{B}}$ distribution reduces to the $\cos \Theta^{*}$ distribution:

$$
\frac{\mathrm{d} N}{\mathrm{~d} \mathcal{C}_{\mathrm{B}}} \underset{\eta^{*} \rightarrow \infty}{\longrightarrow} \frac{\mathrm{d} N}{\mathrm{~d} \cos \Theta^{*}}
$$

This fact served as the original motivation for introducing the $\mathcal{C}_{\mathrm{B}}$ variable in the first place [8]. It is well known (see section 6.2 below) that the $\cos \Theta^{*}$ distribution is directly probing the spins of the particles $A$ and $B$, and so $\mathcal{C}_{\mathrm{B}}$ will inherit some spin sensitivity through (5.10).

The correlation (5.9) is pictorially illustrated in figure 6, which shows scatter plots of $\mathcal{C}_{\mathrm{B}}$ versus $\cos \Theta^{*}$ for several different boost factors $\eta^{*}$ for the two study points from table 3. We see that (5.9) holds only as long as $\sqrt{s}$ is sufficiently large. As $\sqrt{s}$ gets closer to threshold, the correlation is lost and so is the sensitivity of the $\mathcal{C}_{\mathrm{B}}$ variable to the spin effects encoded in the $\cos \Theta^{*}$ distribution (this effect was already evident in figure 4). 
Given that in the off-shell case $\left(A^{*}\right)$ most events at a hadron collider are produced at threshold, we now turn to the opposite limit of $\sqrt{s} \sim \sqrt{s}_{t h}$ or equivalently, $\eta^{*} \rightarrow 0$. In that case, the angle $\Theta^{*}$ becomes arbitrary and can be set to zero, so that (5.6) and (5.7) give

$$
\mathcal{C}_{\mathrm{B}} \underset{\eta^{*} \rightarrow 0, \Theta^{*} \rightarrow 0}{\longrightarrow} \tanh \left(\frac{\eta_{1}^{\prime}-\eta_{2}^{\prime}}{2}\right) \text {. }
$$

With this simple result, one can now perform the integrations in (5.3) and obtain the unit-normalized $\mathcal{C}_{\mathrm{B}}$ distribution at threshold as

$$
\frac{\mathrm{d} N}{\mathrm{~d} \mathcal{C}_{\mathrm{B}}} \underset{\eta^{*} \rightarrow 0}{\longrightarrow} \frac{1-\mathcal{C}_{\mathrm{B}}^{2}}{4 \mathcal{C}_{\mathrm{B}}^{3}}\left\{-2 \mathcal{C}_{\mathrm{B}}+\left(1+\mathcal{C}_{\mathrm{B}}^{2}\right) \ln \left[\frac{1+\mathcal{C}_{\mathrm{B}}}{1-\mathcal{C}_{\mathrm{B}}}\right]\right\} .
$$

This formula is one of the main new results in this paper. It provides the benchmark shape of the $\mathcal{C}_{\mathrm{B}}$ distribution in the vicinity of the energy regime which is relevant for the off-shell case $M_{A}<2 M_{B}$. Eq. (5.12) will be central to our understanding of the effects of spin correlations later on in section 6. Of course, (5.12) is nothing but a crude approximation to the true $\mathcal{C}_{\mathrm{B}}$ distribution which is obtained by integrating over all energies $\sqrt{s}$ as in (5.5). Nevertheless, for practical purposes (5.12) appears to be a more relevant limit than (5.10), since the typical values of $\sqrt{s}$ are closer to threshold than to $\infty$ - see figure 3 .

\subsection{Azimuthal angular difference $\Delta \varphi$}

The azimuthal angular difference $\Delta \varphi$ defined in section 3.2 is given by

$$
\cos \Delta \varphi=\frac{\cos \Delta \phi^{\prime}+D_{0}}{D_{1} D_{2}}
$$

where

$$
\begin{aligned}
D_{0} \equiv & \sin ^{2} \Theta^{*}\left(\cos \phi_{1}^{\prime} \cos \phi_{2}^{\prime}-\sinh \bar{\eta}_{1}^{\prime} \sinh \bar{\eta}_{2}^{\prime}\right) \\
& -\frac{1}{2} \sin 2 \Theta^{*}\left(\cos \phi_{2}^{\prime} \sinh \bar{\eta}_{1}^{\prime}-\cos \phi_{1}^{\prime} \sinh \bar{\eta}_{2}^{\prime}\right), \\
D_{1} \equiv & \left\{1+\sin ^{2} \Theta^{*}\left(\sinh ^{2} \bar{\eta}_{1}^{\prime}-\cos ^{2} \phi_{1}^{\prime}\right)-\sin 2 \Theta^{*} \cos \phi_{1}^{\prime} \sinh \bar{\eta}_{1}^{\prime}\right\}^{1 / 2}, \\
D_{2} \equiv & \left\{1+\sin ^{2} \Theta^{*}\left(\sinh ^{2} \bar{\eta}_{2}^{\prime}-\cos ^{2} \phi_{2}^{\prime}\right)+\sin 2 \Theta^{*} \cos \phi_{2}^{\prime} \sinh \bar{\eta}_{2}^{\prime}\right\}^{1 / 2},
\end{aligned}
$$

and

$$
\bar{\eta}_{1}^{\prime} \equiv \eta^{*}+\eta_{1}^{\prime}, \quad \bar{\eta}_{2}^{\prime} \equiv \eta^{*}-\eta_{2}^{\prime}
$$

Now in the infinite energy limit we obtain

$$
\Delta \varphi \underset{\eta^{*} \rightarrow \infty}{\longrightarrow} \pi
$$

and the universal result

$$
\frac{\mathrm{d} N}{\mathrm{~d} \Delta \varphi} \underset{\eta^{*} \rightarrow \infty}{\longrightarrow} \delta(\Delta \varphi-\pi) .
$$

In the other $\sqrt{s}$ extreme, at threshold, we obtain

$$
\Delta \varphi \underset{\eta^{*} \rightarrow 0}{\longrightarrow} \Delta \phi^{\prime}
$$


and a flat distribution

$$
\frac{\mathrm{d} N}{\mathrm{~d} \Delta \varphi} \underset{\eta^{*} \rightarrow 0}{\longrightarrow} \frac{1}{\pi}=\text { const. }
$$

We see that as $\sqrt{s}$ varies between its two extremes, the $\Delta \varphi$ distribution changes from being completely flat to a perfect delta-function peak at $\pi$. Therefore, the flatness of the $\Delta \varphi$ distribution is a measure of the typical $\sqrt{s}$ in the event, which in turn is affected by spins as shown in figure 3 . Therefore one might expect that the $\Delta \varphi$ distribution would also be sensitive to spin correlations through $\sqrt{s}$ effects, and this is indeed what we saw previously in figures 2,4 and 5 .

\subsection{Invariant mass $M_{\ell^{+} \ell^{-}}$}

On our notation, the formula for the rescaled invariant mass variable (3.7) is

$$
\hat{M}_{\ell^{+} \ell^{-}}=\sqrt{\frac{\cosh \left(\bar{\eta}_{1}^{\prime}+\bar{\eta}_{2}^{\prime}\right)-\cos \left(\phi_{1}^{\prime}-\phi_{2}^{\prime}\right)}{2 \cosh \eta_{1}^{\prime} \cosh \eta_{2}^{\prime}}} .
$$

The integrations in (5.3) can be performed for any fixed $\sqrt{s}>2 M_{B}$ and the result for the unit-normalized $\hat{M}_{\ell^{+} \ell^{-}}$distribution is [36]

$$
\frac{\mathrm{d} N}{\mathrm{~d} \hat{M}_{\ell^{+} \ell^{-}}}= \begin{cases}\frac{4 \eta^{*}}{\sinh 2 \eta^{*}} \hat{M}_{\ell^{+} \ell^{-}}, & \text {for } \hat{M}_{\ell^{+} \ell^{-}} \leq \hat{M}_{\ell^{+} \ell^{-}}^{\text {cusp }}, \\ \frac{2}{\sinh 2 \eta^{*}} \hat{M}_{\ell^{+} \ell^{-}} \log \left(\frac{\hat{M}_{\ell^{+} \ell^{-}}^{\max }}{\hat{M}_{\ell^{+} \ell^{-}}}\right), & \text {for } \hat{M}_{\ell^{+} \ell^{-}}^{\text {cusp }}<\hat{M}_{\ell^{+} \ell^{-}} \leq \hat{M}_{\ell^{+} \ell^{-}}^{\max },\end{cases}
$$

where

$$
\hat{M}_{\ell^{+} \ell^{-}}^{\text {cusp }}=e^{-\eta^{*}}, \quad \hat{M}_{\ell^{+} \ell^{-}}^{\max }=e^{\eta^{*}} .
$$

For events with a given fixed value of $\sqrt{s}$, the distribution (5.23) exhibits a "cusp", i.e. a non-differentiable point, at $\hat{M}_{\ell^{+} \ell^{-}}=\hat{M}_{\ell^{+} \ell^{-}}^{\text {cusp }}$ [36]. However, for $M_{A}<2 M_{B}$, events at hadron colliders occur over a range of values of $\sqrt{s}$ (see figure 3 ) and the cusp gets smeared, since its location depends on the boost factor $\eta^{*}$ and thus on $\sqrt{s} .^{5}$

\subsection{Doubly projected contransverse mass $M_{\mathrm{CT} x}$}

In the absence of spin correlations, the analytical formula for the $M_{\mathrm{CT} x}$ distribution (restricted to events with non-vanishing $M_{\mathrm{CT} x}$ ) is given by [43]

$$
\frac{\mathrm{d} N}{\mathrm{~d} M_{\mathrm{CT} x}}=-4 M_{\mathrm{CT} x} \ln \left(\frac{M_{\mathrm{CT} x}}{M_{\mathrm{CT} x}^{(\max )}}\right) .
$$

Interestingly, this formula does not carry any explicit dependence on $\sqrt{s}$, which can be traced back to the invariance of the $M_{\mathrm{CT} x}$ variable under back-to-back transverse boosts of the parents $B_{1}$ and $B_{2}[43,57]$. Even more peculiar is the observation made in figures 2,4 and 5: that the shape of the $M_{\mathrm{CT} x}$ distribution appears to be pretty much independent of the spins and the mass spectrum in the model. One may therefore wonder whether the

\footnotetext{
${ }^{5}$ Note that in the case of an on-shell $A$ production $\left(M_{A}>2 M_{B}\right)$ events are produced at a fixed $\sqrt{s} \sim M_{A}$ and the cusp has been shown to be experimentally observable [36].
} 

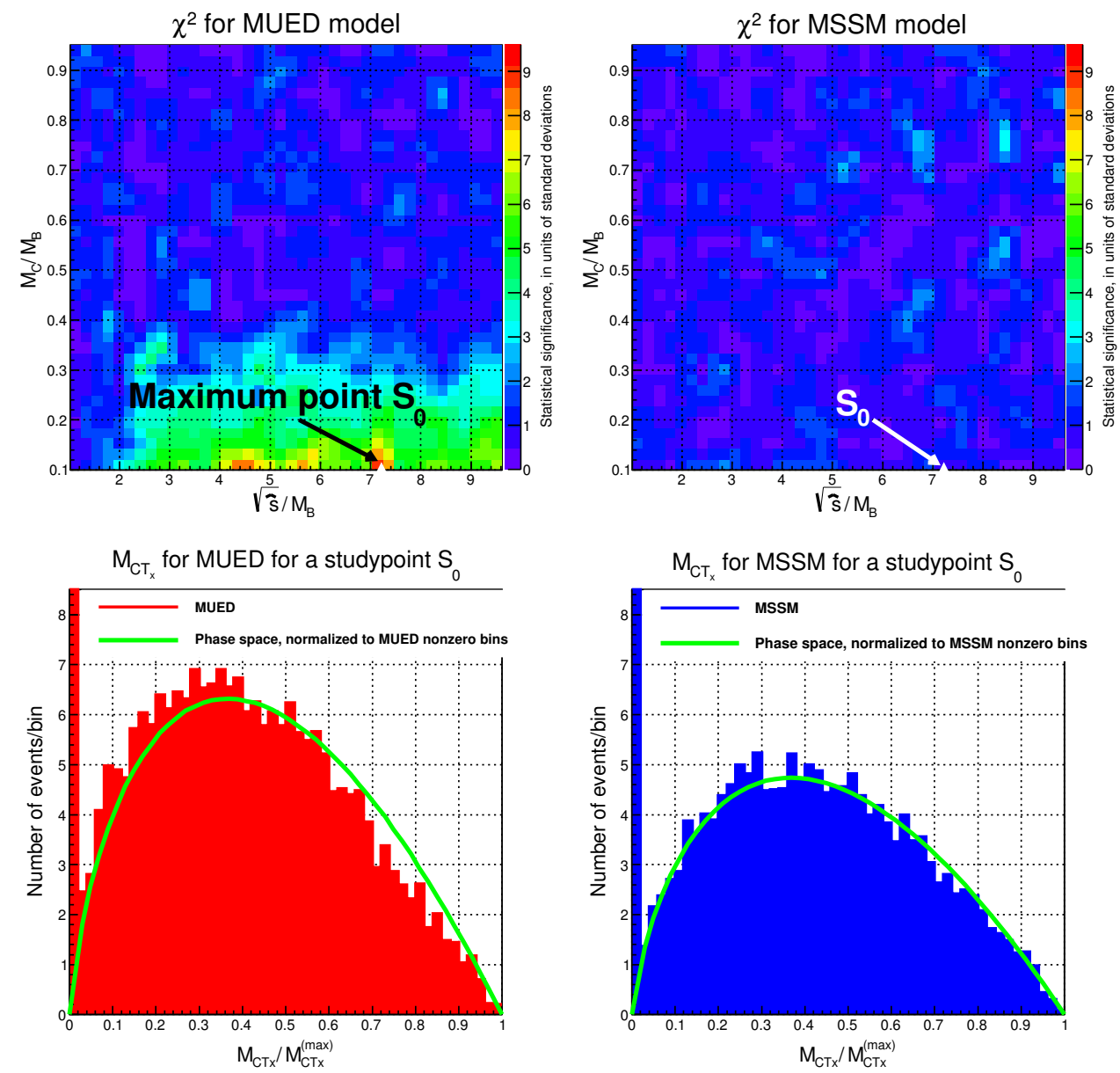

Figure 7. Top: $\chi^{2}$ tests of the predicted $M_{\mathrm{CT} x}$ shape (5.25) against simulated data in MUED (left) and SUSY (right) for a fixed $M_{B}=500 \mathrm{GeV}$ and varying $\sqrt{s}$ and $M_{C} / M_{B}$. Bottom: the $M_{\mathrm{CT} x}$ distributions for the study point $S_{0}$ with the largest $\chi^{2}$.

distribution (5.25) is indeed a universal function which is completely independent of the spin and the kinematics.

In order to address this question, in figure 7 we test how well the formula (5.25) fits the $M_{\mathrm{CT} x}$ distributions in the MUED and SUSY spin scenarios, over a wide range of mass spectra. The upper two panels perform a $\chi^{2}$ test of the predicted shape (5.25) against the $M_{\mathrm{CT} x}$ distribution obtained in our simulations for MUED (left panel) and SUSY (right panel). The test is repeatedly performed for various fixed values of $\sqrt{s}$ (plotted on the $x$-axis) and mass hierarchies $M_{C} / M_{B}$ (plotted on the $y$-axis), for a fixed $M_{B}=500 \mathrm{GeV}$. We see that in the MSSM, the $M_{\mathrm{CT} x}$ shape is very well predicted by (5.25) in all cases. This could have been expected because of the scalar nature of the intermediate particles $B$, which causes the two leptons to be uncorrelated. The formula (5.25) works very well in MUED as well, and only for $M_{C} \ll M_{B}$ one starts to see deviations. In order to illustrate the size of the deviations, in the lower two panels of figure 7 we plot the $M_{\mathrm{CT} x}$ 
distributions for the point $S_{0}$ with the largest $\chi^{2}$ in the MUED case: $M_{C}=0.1 M_{B}$ and $\sqrt{s}=14.4 M_{B}$. We see that even in the worst case scenario of $S_{0}$, the two distributions are in reasonable agreement. We conclude that (5.25) is not a universal function and the shape of the $M_{\mathrm{CT} x}$ variable in principle does get affected by spin correlations, although the effects are very minor.

\subsection{Lepton energy $E_{\ell}$}

The distribution of the lepton energy $E_{\ell}$ has the famous box-like shape for any given fixed $\sqrt{s}$ :

$$
\frac{\mathrm{d} N}{\mathrm{~d} \hat{E}_{\ell}}= \begin{cases}\frac{1}{\hat{E}_{\ell}^{(\max )}-\hat{E}_{\ell}^{(\min )},} & \text { if } \hat{E}_{\ell}^{(\min )} \leq \hat{E}_{\ell} \leq \hat{E}_{\ell}^{(\max )} \\ 0, & \text { otherwise }\end{cases}
$$

where $\hat{E}_{\ell}$ is the rescaled energy variable from (3.17). The two endpoints of the distribution (5.26) are given by

$$
\hat{E}_{\ell}^{(\min )}=e^{-\eta^{*}}, \quad \hat{E}_{\ell}^{(\max )}=e^{\eta^{*}} .
$$

In the threshold limit of $\sqrt{s} \rightarrow 2 M_{B}$ or $\eta^{*} \rightarrow 0$, (5.26) becomes simply

$$
\frac{\mathrm{d} N}{\mathrm{~d} \hat{E}_{\ell}} \underset{\eta^{*} \rightarrow 0}{\longrightarrow} \delta\left(\hat{E}_{\ell}-1\right)
$$

The observation that the two lepton energies are equal and constant at threshold was the main inspiration for the razor class of variables [66]. At hadron colliders, where $\sqrt{s}$ is varying from one event to another, the distribution (5.26) needs to be convoluted as in (5.5) and the two endpoints (5.27) become smeared, as can be seen in figure 2.

The distributions of the remaining four variables from section $3\left(M_{\mathrm{CT}}, M_{\mathrm{eff}}, \sqrt{s}_{\text {min }}\right.$ and $M_{T 2}$ ) do not have compact analytical expressions and we shall not discuss them here.

\section{Spin correlations in the $\mathcal{C}_{\mathrm{B}}$ distribution}

In this section, we shall focus on the $\mathcal{C}_{\mathrm{B}}$ variable and investigate how spin correlations affect the shape of its distribution. In section 5.1 we saw that even in the pure phase space approximation (with no spin effects), we managed to obtain analytical formulas in closed form only for the two limiting cases of $\sqrt{s}$ at threshold and at infinity. Correspondingly, we shall now study the effect of spin correlations on $\mathcal{C}_{\mathrm{B}}$ in those two limits as well.

\subsection{Spin effects at threshold}

We begin with the threshold limit $\sqrt{s} \sim \sqrt{s}_{t h}\left(=2 M_{B}\right)$, where the $\mathcal{C}_{\mathrm{B}}$ distribution is given by the previously derived formula (5.12). Then we ask, how will the presence of spin correlations modify the phase space result (5.12). For this purpose, in appendix A we rederive the unit-normalized $\mathcal{C}_{\mathrm{B}}$ distributions at threshold for each of the 8 spin scenarios 
in table 1, and for arbitrary values of the relative chirality parameters $\alpha, \beta$ and $\gamma$ defined in $(2.12)-(2.14)$. The result is

$$
\begin{aligned}
& \left.\frac{\mathrm{d} N}{\mathrm{~d} \mathcal{C}_{\mathrm{B}}}\right|_{\mathbf{T H}} ^{\mathrm{SSF}}=\mathrm{J}_{\mathrm{PS}}, \\
& \left.\frac{\mathrm{d} N}{\mathrm{~d} \mathcal{C}_{\mathrm{B}}}\right|_{\mathbf{T H}} ^{\mathrm{SFS}}=\mathrm{J}_{\mathrm{PS}}+\frac{\gamma^{2}}{4} \cdot \mathrm{J}_{\mathrm{F}}, \\
& \left.\frac{\mathrm{d} N}{\mathrm{~d} \mathcal{C}_{\mathrm{B}}}\right|_{\mathbf{T H}} ^{\mathrm{SFV}}=\mathrm{J}_{\mathrm{PS}}+\frac{\gamma^{2}}{4}\left(\frac{1-2 y}{1+2 y}\right)^{2} \cdot \mathrm{J}_{\mathrm{F}}, \\
& \left.\frac{\mathrm{d} N}{\mathrm{~d} \mathcal{C}_{\mathrm{B}}}\right|_{\mathbf{T H}} ^{\mathrm{SVF}}=\frac{3(1+2 y)}{(2+y)^{2}}\left\{\mathrm{~J}_{\mathrm{PS}}+\frac{(1-y)^{2}}{4(1+2 y)} \cdot \mathrm{J}_{\mathrm{V}}-\frac{\gamma^{2}}{2(1+2 y)} \cdot \mathrm{J}_{\mathrm{F}}\right\}, \\
& \left.\frac{\mathrm{d} N}{\mathrm{~d} \mathcal{C}_{\mathrm{B}}}\right|_{\mathbf{T H}} ^{\mathbf{V S F}}=\mathrm{J}_{\mathrm{PS}}, \\
& \left.\frac{\mathrm{d} N}{\mathrm{~d} \mathcal{C}_{\mathrm{B}}}\right|_{\mathbf{T H}} ^{\mathbf{V F S}}=\mathrm{J}_{\mathrm{PS}}-\frac{\gamma^{2}}{4} \cdot \mathrm{J}_{\mathrm{F}}, \\
& \left.\frac{\mathrm{d} N}{\mathrm{~d} \mathcal{C}_{\mathrm{B}}}\right|_{\mathbf{T H}} ^{\mathbf{V F V}}=\mathrm{J}_{\mathrm{PS}}-\frac{\gamma^{2}}{4}\left(\frac{1-2 y}{1+2 y}\right)^{2} \cdot \mathrm{J}_{\mathrm{F}}, \\
& \left.\frac{\mathrm{d} N}{\mathrm{~d} \mathcal{C}_{\mathrm{B}}}\right|_{\mathbf{T H}} ^{\mathbf{V V F}}=\frac{9(1+y)}{2(2+y)^{2}}\left\{\mathrm{~J}_{\mathrm{PS}}-\frac{(1-y)^{2}}{4(1+y)} \cdot \mathrm{J}_{\mathrm{V} 1}-\frac{y(1-y)}{8(1+y)} \cdot \mathrm{J}_{\mathrm{V} 2}\right\},
\end{aligned}
$$

where

$$
y \equiv \frac{M_{C}^{2}}{M_{B}^{2}}
$$

and the basis functions $\mathrm{J}_{i}$ are defined as follows,

$$
\begin{aligned}
\mathrm{J}_{\mathrm{PS}}= & \frac{1-\mathcal{C}_{\mathrm{B}}^{2}}{4 \mathcal{C}_{\mathrm{B}}^{3}}\left\{-2 \mathcal{C}_{\mathrm{B}}+\left(1+\mathcal{C}_{\mathrm{B}}^{2}\right) \ln \left[\frac{1+\mathcal{C}_{\mathrm{B}}}{1-\mathcal{C}_{\mathrm{B}}}\right]\right\} \\
\mathrm{J}_{\mathrm{F}}= & \frac{1-\mathcal{C}_{\mathrm{B}}^{2}}{4 \mathcal{C}_{\mathrm{B}}^{5}}\left\{6 \mathcal{C}_{\mathrm{B}}+4 \mathcal{C}_{\mathrm{B}}^{3}+6 \mathcal{C}_{\mathrm{B}}^{5}-\left(3+\mathcal{C}_{\mathrm{B}}^{2}+\mathcal{C}_{\mathrm{B}}^{4}+3 \mathcal{C}_{\mathrm{B}}^{6}\right) \ln \left(\frac{1+\mathcal{C}_{\mathrm{B}}}{1-\mathcal{C}_{\mathrm{B}}}\right)\right\} \\
\mathrm{J}_{\mathrm{V}}= & \frac{1-\mathcal{C}_{\mathrm{B}}^{2}}{48 \mathcal{C}_{\mathrm{B}}^{7}} \cdot\left\{-2 \mathcal{C}_{\mathrm{B}}\left(45+22 \mathcal{C}_{\mathrm{B}}^{4}+45 \mathcal{C}_{\mathrm{B}}^{8}\right)\right. \\
& \left.+3\left(15-5 \mathcal{C}_{\mathrm{B}}^{2}+6 \mathcal{C}_{\mathrm{B}}^{4}+6 \mathcal{C}_{\mathrm{B}}^{6}-5 \mathcal{C}_{\mathrm{B}}^{8}+15 \mathcal{C}_{\mathrm{B}}^{10}\right) \ln \left(\frac{1+\mathcal{C}_{\mathrm{B}}}{1-\mathcal{C}_{\mathrm{B}}}\right)\right\} \\
\mathrm{J}_{\mathrm{V} 1}= & \frac{1-\mathcal{C}_{\mathrm{B}}^{2}}{24 \mathcal{C}_{\mathrm{B}}^{7}} \cdot\left\{-2 \mathcal{C}_{\mathrm{B}}\left(15+8 \mathcal{C}_{\mathrm{B}}^{2}+10 \mathcal{C}_{\mathrm{B}}^{4}+8 \mathcal{C}_{\mathrm{B}}^{6}+15 \mathcal{C}_{\mathrm{B}}^{8}\right)\right. \\
& \left.+3\left(5+\mathcal{C}_{\mathrm{B}}^{2}+2 \mathcal{C}_{\mathrm{B}}^{4}+2 \mathcal{C}_{\mathrm{B}}^{6}+\mathcal{C}_{\mathrm{B}}^{8}+5 \mathcal{C}_{\mathrm{B}}^{10}\right) \ln \left(\frac{1+\mathcal{C}_{\mathrm{B}}}{1-\mathcal{C}_{\mathrm{B}}}\right)\right\} \\
\mathrm{J}_{\mathrm{V} 2}= & \frac{1}{3 \mathcal{C}_{\mathrm{B}}^{5}}\left\{-2 \mathcal{C}_{\mathrm{B}}\left(3+\mathcal{C}_{\mathrm{B}}^{2}-\mathcal{C}_{\mathrm{B}}^{4}-3 \mathcal{C}_{\mathrm{B}}^{6}\right)+3\left(1-\mathcal{C}_{\mathrm{B}}^{8}\right) \ln \left(\frac{1+\mathcal{C}_{\mathrm{B}}}{1-\mathcal{C}_{\mathrm{B}}}\right)\right\}
\end{aligned}
$$




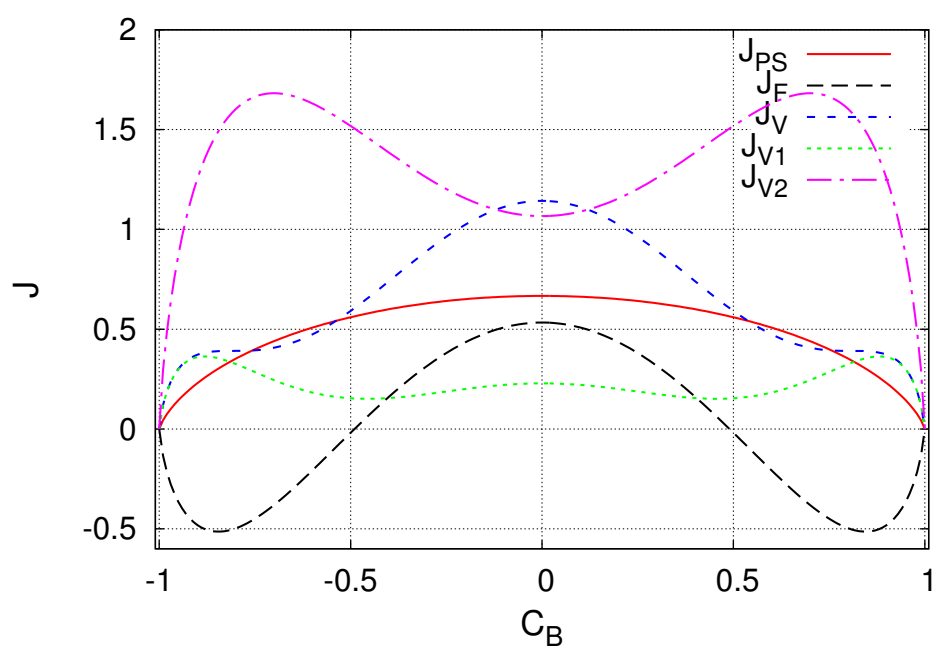

Figure 8. The basis functions $\mathrm{J}\left(\mathcal{C}_{\mathrm{B}}\right)$ defined in $(6.10)-(6.14)$. The solid red line is the pure phase space distribution (6.10) or equivalently (5.12).

The basis functions (6.10)-(6.14) are pictorially illustrated in figure 8 . The function $\mathrm{J}_{\mathrm{PS}}$ is simply the phase space limit (5.12) already derived in section 5.1, while the remaining 4 basis functions parameterize the different possible distortions of the pure phase space shape, for the various spin scenarios.

Formulas (6.1)-(6.8) represent one of our main results. Using (6.1)-(6.8), one can understand how the shape of the $\mathcal{C}_{\mathrm{B}}$ distribution near threshold changes as a function of the mass spectrum (through the dependence on the $y$ parameter) and as a function of the relative chirality parameter $\gamma$ defined in (2.12). (The parameters $\alpha$ and $\beta$ decouple and do not enter the threshold distribution formulas.) For example, (6.1) and (6.5) show that in the cases where the intermediate particles $B$ are scalars, any spin correlations between the two leptons are wiped out and one obtains the pure phase space shape (6.10), which peaks at $\mathcal{C}_{\mathrm{B}}=0$ and vanishes at $\mathcal{C}_{\mathrm{B}}= \pm 1$ (see the solid red line in figure 8 ). Furthermore, the predictions (6.1) and (6.5) are not sensitive to the mass spectrum at all, since the mass ratio $y$ does not enter those formulas.

Now let us proceed to the SFS and VFS scenarios of (6.2) and (6.6), correspondingly. Here we find that the spin correlations induce a second term involving the $\mathrm{J}_{\mathrm{F}}$ function, which has a maximum at $\mathcal{C}_{\mathrm{B}}=0$ and two local minima at $\mathcal{C}_{\mathrm{B}}= \pm 0.845$ (see the black dashed line in figure 8). The coefficient of the $J_{F}$ term is positive in (6.2) and negative in (6.6). This means that in the SFS spin scenario, the addition of spin correlations will make the central peak of the pure phase space distribution $\mathrm{J}_{\mathrm{PS}}$ even more pronounced. This is illustrated in the upper left panel of figure 9 , where we start with the pure phase space distribution at $\gamma=0$ (the black solid line) and gradually increase the amount of spin correlations until we get purely chiral couplings at $\gamma=1$. As expected, the presence of spin correlations in the SFS scenario makes the central peak in the $\mathcal{C}_{\mathrm{B}}$ distribution steeper. In the VFS scenario, on the other hand, spin correlations have just the opposite effect, since 

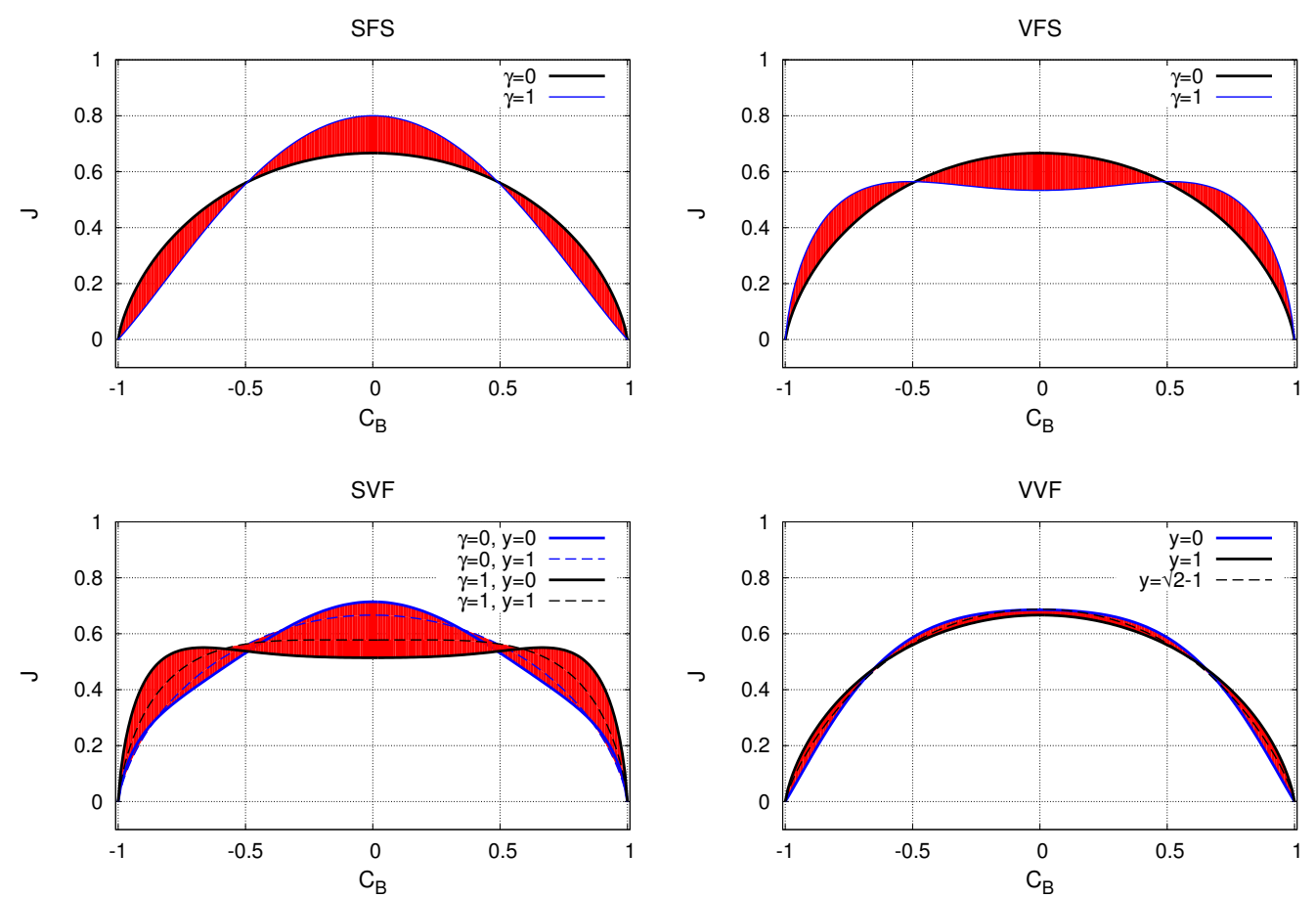

Figure 9. Spin correlation effects on the $\mathcal{C}_{\mathrm{B}}$ distribution at threshold, for various spin scenarios. In each case, we start with the pure phase space distribution at $\gamma=0$ and/or $y=1$, and show the amount of shape distortion by varying $\gamma$ and $y$ over their full range.

the $J_{F}$ term in (6.6) comes with a minus sign. Now, the central peak will be suppressed, and events will shift towards the two endpoints instead. The resulting shape distortion is illustrated in the upper right panel of figure 9, where again we show the full range from $\gamma=0$ (pure phase space) to $\gamma=1$ (purely chiral couplings). We see that the presence of chiral couplings causes the VFS distribution to be much flatter than in the pure phase space limit.

The SFS and VFS results shown in the upper two panels of figure 9 are also independent of the mass spectrum, as the mass parameter $y$ does not enter eqs. (6.2) and (6.6). In contrast, the remaining four spin cases to be discussed now will exhibit a dependence on the mass spectrum through $y$. It is probably easiest to start with the SFV and VFV spin configurations, whose $\mathcal{C}_{\mathrm{B}}$ distributions are given by (6.3) and (6.7), respectively. We find very similar behavior to the two cases of SFS and VFS just discussed - the only difference is that the coefficient of the $\mathrm{J}_{\mathrm{F}}$ function is now additionally suppressed by a factor of $(1-2 y)^{2} /(1+2 y)^{2}$, which incorporates the mass spectrum dependence. Because of this suppression, one would generally expect smaller variations than what we saw in the upper two panels of figure 9 . There even exists a very special case with $y=1 / 2$ (i.e. $M_{B}=\sqrt{2} M_{C}$ ) when the $\mathcal{C}_{\mathrm{B}}$ distribution reduces to the pure phase space prediction (6.10). For that particular mass spectrum, the SFV and VFV distributions at threshold would be identical to the SSF and VSF distributions, and spin discrimination would have to be done in a regime away from threshold. 
Let us now move on to the SVF scenario of (6.4). This is perhaps the most complicated case, since in addition to the $\mathrm{J}_{\mathrm{PS}}$ baseline shape it has two extra terms - one with the already familiar function $\mathrm{J}_{\mathrm{F}}$, and a second term involving the function $\mathrm{J}_{\mathrm{V}}$ ( shown in figure 8 with the blue dashed line). The function $\mathrm{J}_{\mathrm{V}}$ peaks at $\mathcal{C}_{\mathrm{B}}=0$ and has broad shoulders near the endpoints of the $\mathcal{C}_{\mathrm{B}}$ interval. The coefficient of $\mathrm{J}_{\mathrm{V}}$ in (6.4) is always positive, which means that the $J_{V}$ term will always tend to enhance the central peak. At the same time, the coefficient of the $J_{F}$ term in (6.4) is always negative, which would tend to suppress the central peak, as already seen in the upper right panel of figure 9. Thus the interplay of the two terms will lead to partial cancellations, which are illustrated in the lower left panel of figure 9. The dashed blue line represents the pure phase space limit in eq. (6.4), which is recovered when $\gamma=0$ and $y=1$. If we keep $\gamma=0$, the $\mathrm{J}_{\mathrm{F}}$ term is turned off, and by varying $y$, we can see the impact of the $\mathrm{J}_{\mathrm{V}}$ term in (6.4), which is maximal at $y=0$ (the solid blue line). Conversely, if we keep $y=1$, the $\mathrm{J}_{\mathrm{V}}$ term is turned off, and the variations in $\gamma$ reveal the effect of the $\mathrm{J}_{\mathrm{F}}$ term, which is maximal at $\gamma=1$ (the red dashed line). The case when both terms are maximal $(\gamma=1$ and $y=0)$ is given by the solid black line.

Finally, we discuss the VVF case of eq. (6.8), pictured in the lower right panel of figure 9 . Here the $\mathcal{C}_{\mathrm{B}}$ distribution at threshold depends only on the mass spectrum but not on the chirality parameter $\gamma$. The spin correlation effects are encoded in the two functions $\mathrm{J}_{\mathrm{V} 1}$ and $\mathrm{J}_{\mathrm{V} 2}$, represented in figure 8 by the green dotted and magenta dot-dashed lines, respectively. $\quad \mathrm{J}_{\mathrm{V} 1}$ has local maxima at $\mathcal{C}_{\mathrm{B}}=0$ and $\mathcal{C}_{\mathrm{B}}= \pm 0.881$ and local minima at $\mathcal{C}_{\mathrm{B}}= \pm 0.456$, while $\mathrm{J}_{\mathrm{V} 2}$ has local maxima at $\mathcal{C}_{\mathrm{B}}= \pm 0.7$ and a local minimum at $\mathcal{C}_{\mathrm{B}}=0$. The lower right panel in figure 9 shows the resulting variation in the $\mathcal{C}_{\mathrm{B}}$ shape as we vary $y$ from $y=1$ (the pure phase space limit) to $y=0$ (when the $\mathrm{J}_{\mathrm{V} 1}$ term is maximal). We see only very minor variations, due to the fact that the coefficients of both the $J_{\mathrm{V} 1}$ and $\mathrm{J}_{\mathrm{V} 2}$ terms in (6.8) are numerically very small.

This concludes our discussion of eqs. (6.1)-(6.8), which manifestly describe the spin effects at threshold. As figure 3 showed, although most events are produced near threshold, virtually none are produced exactly at threshold, thus eqs. (6.1)-(6.8) are perhaps of limited practical interest. Their true value is in developing some intuition about the shape of the $\mathcal{C}_{\mathrm{B}}$ distribution, which could prove useful for the interpretation of the finite $\sqrt{s}$ results below.

\subsection{Spin effects in the large energy limit}

We now turn our attention to the other $\sqrt{s}$ extreme, namely $\sqrt{s} \rightarrow \infty$. In that case, as discussed in section 5.1 and shown in $(5.10)$, the $\mathcal{C}_{\mathrm{B}}$ distribution reduces to the $\cos \Theta^{*}$ distribution for $2 \rightarrow 2$ processes, whose dependence on the spins of the particles is well known, so we have

$$
\begin{aligned}
& \left.\frac{\mathrm{d} N}{\mathrm{~d} \mathcal{C}_{\mathrm{B}}}\right|_{\infty} ^{\mathrm{SXY}} \propto 1, \quad \text { for }\{X, Y\} \subset\{\mathrm{S}, \mathrm{F}, \mathrm{V}\}, \\
& \left.\frac{\mathrm{d} N}{\mathrm{~d} \mathcal{C}_{\mathrm{B}}}\right|_{\infty} ^{\mathbf{V F Y}} \propto 1+\mathcal{C}_{\mathrm{B}}^{2}, \quad \text { for } Y \in\{\mathrm{S}, \mathrm{V}\}, \\
& \left.\frac{\mathrm{d} N}{\mathrm{~d} \mathcal{C}_{\mathrm{B}}}\right|_{\infty} ^{\mathbf{V Y F}} \propto 1-\mathcal{C}_{\mathrm{B}}^{2}, \quad \text { for } Y \in\{\mathrm{S}, \mathrm{V}\} .
\end{aligned}
$$


These equations predict three generic shapes for the $\mathcal{C}_{\mathrm{B}}$ distribution at large energies:

- Flat as in (6.15). This is the case whenever $A$ is a scalar particle.

- $1+\mathcal{C}_{\mathrm{B}}^{2}$ as in (6.16). This case occurs when $A$ has spin 1 and $B$ is a fermion, e.g. the UED example from section 3 falls into this category. The distribution (6.16) peaks at $\mathcal{C}_{\mathrm{B}}= \pm 1$ and has a minimum at $\mathcal{C}_{\mathrm{B}}=0$.

- $1-\mathcal{C}_{\mathrm{B}}^{2}$ as in (6.17). This is the case when $A$ has spin 1 and $B$ is a boson, as in the SUSY example considered earlier. The $\mathcal{C}_{\mathrm{B}}$ distribution then has a peak at $\mathcal{C}_{\mathrm{B}}=0$ and vanishes at the endpoints $\mathcal{C}_{\mathrm{B}}= \pm 1$.

As shown in section 5.1, the nice correlation (5.10) between $\mathcal{C}_{\mathrm{B}}$ and $\cos \Theta^{*}$ emerges only at sufficiently large energies, which might not be realistically achieved at a hadron collider. Therefore, the practical value of the limits (6.15)-(6.17) is also debatable, just like the formulas (6.1)-(6.8) from section 6.1. However, with the use of (6.1)-(6.8) and (6.15)(6.17), one can begin to understand the observed $\mathcal{C}_{\mathrm{B}}$ shapes in figure 2 , where we noticed that the MUED case gives a flatter $\mathcal{C}_{\mathrm{B}}$ distribution than the MSSM. In the case of the MSSM, the $\mathcal{C}_{\mathrm{B}}$ distribution at threshold (6.5) peaks at $\mathcal{C}_{\mathrm{B}}=0$ and vanishes at $\mathcal{C}_{\mathrm{B}}= \pm 1$, while the large energy limit (6.17) also peaks at $\mathcal{C}_{\mathrm{B}}=0$ and vanishes at $\mathcal{C}_{\mathrm{B}}= \pm 1$. The $\mathcal{C}_{\mathrm{B}}$ distribution at finite $\sqrt{s}$ would then interpolate between those two very similar limiting cases and inherit their common properties - which explains why the actual MSSM distribution in figure 2 has a relatively sharp peak at $\mathcal{C}_{\mathrm{B}}=0$ and vanishes at $\mathcal{C}_{\mathrm{B}}= \pm 1$.

In the case of MUED, we have a different story - here at intermediate $\sqrt{s}$ we have to interpolate between two very different shapes: the threshold distribution (6.7) which typically peaks at $\mathcal{C}_{\mathrm{B}}=0$ and vanishes at $\mathcal{C}_{\mathrm{B}}= \pm 1$, and the asymptotic distribution (6.16) which peaks at $\mathcal{C}_{\mathrm{B}}= \pm 1$ and has a minimum at $\mathcal{C}_{\mathrm{B}}=0$. At the end of the day, the resulting $\mathcal{C}_{\mathrm{B}}$ shape will depend on the exact form of the underlying $\sqrt{s}$ distribution, but figure 3 suggests that the threshold behavior will dominate, since a typical event is produced closer to threshold. This is indeed what we observe in figure 2 , where the $\mathcal{C}_{\mathrm{B}}$ distribution in the MUED case also peaks at $\mathcal{C}_{\mathrm{B}}=0$, in spite of the asymptotic expectation (6.16).

\subsection{Spin effects near threshold}

When particle $A$ is off-shell (i.e. $M_{A}<2 M_{B}$ ), the onset of the $p p \rightarrow B_{1} B_{1}$ production cross-section as a function of $\sqrt{s}$ is sensitive to the spins of $A$ and $B$. The partonic cross section at a given $\sqrt{s}$ can be expressed as

$$
\hat{\sigma}(\sqrt{s})=\frac{1}{2 s} \int \mathrm{d} \Pi_{2}^{*}|M(\sqrt{s})|^{2}=\left(\frac{1}{8 \pi}\right)^{2} \frac{\beta_{\mathrm{CM}}}{s} \int \mathrm{d} \Omega^{*}|M(\sqrt{s})|^{2},
$$

where $\beta_{\mathrm{CM}}$ is the $B_{i}$ boost factor in the CMBB frame

$$
\beta_{\mathrm{CM}} \equiv \tanh \eta^{*} .
$$


The cross-section near threshold is suppressed by a certain power of $\beta_{\mathrm{CM}}$, which depends on the spin scenario:

$$
\begin{aligned}
|M(\sqrt{s})|_{(\mathrm{SS})}^{2} & \propto 1+\mathcal{O}\left(\beta_{\mathrm{CM}}^{2}\right), \\
|M(\sqrt{s})|_{(\mathrm{SF})}^{2} & \propto(1-\cos \delta)+(1+\cos \delta) \beta_{\mathrm{CM}}^{2}+\mathcal{O}\left(\beta_{\mathrm{CM}}^{4}\right), \\
|M(\sqrt{s})|_{(\mathrm{SV})}^{2} & \propto 1+\mathcal{O}\left(\beta_{\mathrm{CM}}^{2}\right), \\
|M(\sqrt{s})|_{(\mathrm{VS})}^{2} & \propto \beta_{\mathrm{CM}}^{2}\left(1-\cos ^{2} \Theta^{*}\right)+\mathcal{O}\left(\beta_{\mathrm{CM}}^{4}\right), \\
|M(\sqrt{s})|_{(\mathrm{VF})}^{2} & \propto 1+\mathcal{O}\left(\beta_{\mathrm{CM}}\right), \\
|M(\sqrt{s})|_{(\mathrm{VV})}^{2} & \propto \beta_{\mathrm{CM}}^{2}\left(1-\frac{3}{19} \cos ^{2} \Theta^{*}\right)+\mathcal{O}\left(\beta_{\mathrm{CM}}^{4}\right) .
\end{aligned}
$$

Interestingly, the $S F$ case can be subdivided into two categories: a real scalar

$$
|M(\sqrt{s})|_{\left(\mathrm{S}^{++} \mathrm{F}\right)}^{2} \propto \beta_{\mathrm{CM}}^{2}+\mathcal{O}\left(\beta_{\mathrm{CM}}^{4}\right)
$$

or a pseudo-scalar

$$
|M(\sqrt{s})|_{\left(\mathrm{S}^{-+} \mathrm{F}\right)}^{2} \propto 1+\mathcal{O}\left(\beta_{\mathrm{CM}}^{4}\right)
$$

Thus, the $\beta_{\mathrm{CM}}$ suppression factor near threshold is as follows:

$$
\frac{\mathrm{d} \hat{\sigma}(\sqrt{s})}{\mathrm{d} \Omega^{*}} \propto \begin{cases}\beta_{\mathrm{CM}}, & \text { for }(\mathrm{SS}),\left(\mathrm{S}^{P C} \mathrm{~F}\right)=\left(\mathrm{S}^{-+} \mathrm{F}\right),(\mathrm{SV}),(\mathrm{VF}) \\ \beta_{\mathrm{CM}}^{3}, & \text { for }\left(\mathrm{S}^{P C} \mathrm{~F}\right)=\left(\mathrm{S}^{++} \mathrm{F}\right),(\mathrm{VS}),(\mathrm{VV})\end{cases}
$$

\section{Comparison of the different spin scenarios}

We are now ready to contrast the different spin scenarios and discuss the prospects for spin discrimination. In principle, one could pose two questions:

1. How well can two different spin scenarios be discriminated experimentally, given the uncertainty in the coupling chiralities and instrumental effects like SM backgrounds and the finite detector resolution. Clearly, the answer to this question will depend on many quantitative factors - the chosen study point, the size of the signal, etc., and it is difficult if not impossible to give a "one size fits all" answer.

2. Which spin scenarios (and under what circumstances) are in danger of being confused with each other? This question is easier to tackle theoretically, because if we can identify the cases where two spin scenarios look identical at the parton level, the conclusions will remain unchanged when we add all the usual experimental complications. This is why in this section we shall use the intuition developed in previous sections to pinpoint the difficult cases for spin discrimination.

Let us focus on the $\mathcal{C}_{\mathrm{B}}$ distribution. Table 4 summarizes its salient features identified in section 6: the behavior at threshold (second column) or asymptotically at $\sqrt{s} \rightarrow \infty$ (third column), and the power of any additional $\beta_{\mathrm{CM}}$ threshold suppression arising from 


\begin{tabular}{|c|c|c|c|c|c|}
\hline Configuration & $\sqrt{s} \rightarrow 2 M_{B}$ & $\sqrt{s} \rightarrow \infty$ & \multicolumn{2}{|c|}{$|M|^{2} \propto \beta_{C M}^{n}$} & Evil twin \\
\hline SSF & $\overline{\mathrm{J}_{\mathrm{PS}}}$ & 1 & \multicolumn{2}{|c|}{1} & SVF \\
\hline SVF & $\mathrm{J}_{\mathrm{PS}}+\epsilon_{2} \mathrm{~J}_{\mathrm{V}}-\frac{\gamma^{2}}{4} \epsilon_{3} \mathrm{~J}_{\mathrm{F}}$ & 1 & \multicolumn{2}{|c|}{1} & SSF \\
\hline SFS & $\mathrm{J}_{\mathrm{PS}}+\frac{\gamma^{2}}{4} \mathrm{~J}_{\mathrm{F}}$ & 1 & $\begin{array}{c}\mathrm{S}^{-+} \mathrm{FS} \\
1\end{array}$ & $\begin{array}{c}\mathrm{S}^{++} \mathrm{FS} \\
2\end{array}$ & SFV \\
\hline SFV & $\mathrm{J}_{\mathrm{PS}}+\frac{\gamma^{2}}{4} \epsilon_{1} \mathrm{~J}_{\mathrm{F}}$ & 1 & $\begin{array}{c}\mathrm{S}^{-+} \mathrm{FV} \\
1\end{array}$ & $\begin{array}{c}\mathrm{S}^{++} \mathrm{FV} \\
2\end{array}$ & SFS \\
\hline VSF & $\overline{\mathrm{J}_{\mathrm{PS}}}$ & $1-\mathcal{C}_{\mathrm{B}}^{2}$ & \multicolumn{2}{|c|}{2} & VVF \\
\hline VVF & $\mathrm{J}_{\mathrm{PS}}-\epsilon_{4} \mathrm{~J}_{\mathrm{V} 1}-\epsilon_{5} \mathrm{~J}_{\mathrm{V} 2}$ & $1-\mathcal{C}_{\mathrm{B}}^{2}$ & \multicolumn{2}{|c|}{2} & VSF \\
\hline VFS & $\mathrm{J}_{\mathrm{PS}}-\frac{\gamma^{2}}{4} \mathrm{~J}_{\mathrm{F}}$ & $1+\mathcal{C}_{\mathrm{B}}^{2}$ & \multicolumn{2}{|c|}{1} & VFV \\
\hline VFV & $\mathrm{J}_{\mathrm{PS}}-\frac{\gamma^{2}}{4} \epsilon_{1} \mathrm{~J}_{\mathrm{F}}$ & $1+\mathcal{C}_{\mathrm{B}}^{2}$ & \multicolumn{2}{|c|}{1} & VFS \\
\hline
\end{tabular}

Table 4. The asymptotic behavior of the $\mathcal{C}_{\mathrm{B}}$ distribution at threshold (second column) and at $\sqrt{s} \rightarrow \infty$ (third column) for different spin configurations in the antler topology. The third column lists the power of any additional $\beta_{\mathrm{CM}}$ threshold suppression coming from the matrix element. The last column shows the alternative spin scenario with similar properties.

the matrix element. For convenience, formulas (6.1)-(6.8) are rewritten in terms of the shorthand notation

$$
\begin{aligned}
& \epsilon_{1}=\left(\frac{1-2 y}{1+2 y}\right)^{2} \\
& \epsilon_{2}=\frac{(1-y)^{2}}{4(1+2 y)} \\
& \epsilon_{3}=\frac{2}{(1+2 y)} \\
& \epsilon_{4}=\frac{(1-y)^{2}}{4(1+y)} \\
& \epsilon_{5}=\frac{y(1-y)}{8(1+y)} .
\end{aligned}
$$

In table 4 , the 8 spin configurations from table 1 are arranged in pairs. The two spin scenarios within each pair can exhibit identical behavior both at threshold (for a suitably chosen mass spectrum) and at $\sqrt{s} \rightarrow \infty$, and have the same $\beta_{\mathrm{CM}}$ threshold suppression. Those pairs are therefore the problematic cases where spin discrimination based on $\mathcal{C}_{\mathrm{B}}$ might be difficult. Of course, this is simply a conjecture based on the asymptotic behavior, so it is worth checking if it holds for the $\mathcal{C}_{\mathrm{B}}$ distribution at any $\sqrt{s}$. Then if it turns out that the $\mathcal{C}_{\mathrm{B}}$ distribution is indeed very similar for a given pair of spin configurations, we will then check whether any of the remaining variables discussed in section 3 can offer an alternative tool for discrimination.

Figures 10-14 show the distributions of the nine variables from section 3, for each of the twin spin scenarios in table 4. All simulations were done with MadGraph v4 [67], in the narrow width approximation, for an $8 \mathrm{TeV}$ LHC. In all figures, we consider the case of a heavy resonance $A$ with $M_{A}=1.2 \mathrm{TeV}$, and intermediate particles $B_{i}$ with mass $M_{B}=500 \mathrm{GeV}$. We then adjust the mass of $M_{C}$ appropriately in order to obtain an exact match in the $\mathcal{C}_{\mathrm{B}}$ distributions at threshold. For example, figure 10 compares the case of SSF to SVF. The former has a $\mathcal{C}_{\mathrm{B}}$ distribution which is given by the pure phase space formula at threshold, while the $\mathcal{C}_{\mathrm{B}}$ distribution for the latter involves terms proportional to $\epsilon_{2}$ and $\epsilon_{3} \gamma^{2} / 4$ (see table 4 ). Then the worst case scenario would have a degenerate spectrum with $y \rightarrow 1\left(M_{B} \sim M_{C}\right)$, which would set $\epsilon_{2}=0$, and purely vector-like couplings with 

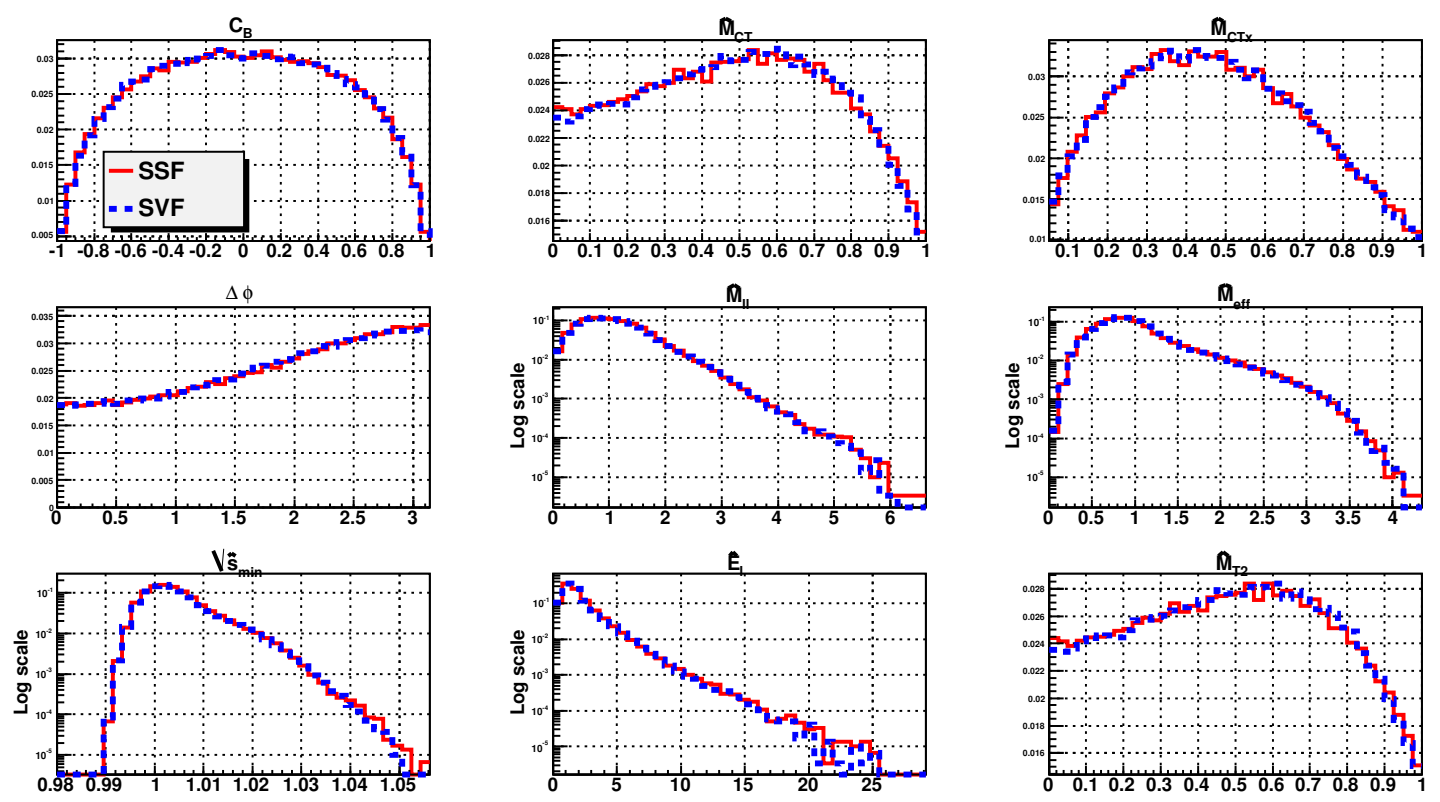

Figure 10. The same as figure 2, but this time comparing kinematic distributions for the SSF and SVF spin scenarios at an $8 \mathrm{TeV}$ LHC. The couplings and masses in each case are the same, and were chosen to match the asymptotic $\mathcal{C}_{\mathrm{B}}$ distribution at threshold $(y \sim 1$ and $\gamma=0): M_{A}=1200 \mathrm{GeV}$, $M_{B}=500 \mathrm{GeV}$ and $M_{C}=495 \mathrm{GeV}$. For this case, $\beta$ is not defined, while the choice of $\alpha$ is inconsequential.

$\gamma=0$. This is precisely the case shown in figure 10, where for definiteness we choose $M_{C}=495 \mathrm{GeV}$. As expected, the two $\mathcal{C}_{\mathrm{B}}$ distributions are almost identical and can hardly be used to measure the spins. The remaining plots in figure 10 then check whether any of the other eight distributions show observable differences. As it turns out, they are all pretty well matched as well, and the spin discrimination between SSF and SVF is indeed very problematic under these circumstances.

In the next two figures we show a similar comparison between the SFS and SFV spin scenarios: in figure 11 for the case when particle $A$ is a scalar $\left(S^{++}\right)$and in figure 12 for the case when particle $A$ is a pseudoscalar $\left(S^{-+}\right)$Here, to match the threshold $\mathcal{C}_{\mathrm{B}}$ distributions, it is sufficient to set $y=0$ (for definiteness, we choose $M_{C}=5 \mathrm{GeV}$ ). Figures 11 and 12 reveal a similarly grim situation - the distributions of all 9 variables match almost perfectly in the two cases, and any spin discrimination thus appears to be virtually impossible.

The next pair of similar spin configurations in table 4 is VSF and VVF, which are compared in figure 13. The threshold $\mathcal{C}_{\mathrm{B}}$ distribution for VSF is given by the pure phase space result (6.10) while for VVF, it reduces to the same formula if $y=1$. In order to mimic the case of $y=1$, we again choose $M_{C}=495 \mathrm{GeV}$. Once again, we find that the distributions of all 9 variables are pretty similar.

The last problematic pair of spin configurations in table 4 is VFS and VFV. Their $\mathcal{C}_{\mathrm{B}}$ distributions at threshold become identical if $\gamma=0$ or if $y \sim 0$. Figure 14 shows a case with $\gamma=0$ and $y \sim 0$. Once again we observe that all 9 kinematic variables behave very similarly and spin discrimination is very difficult. 

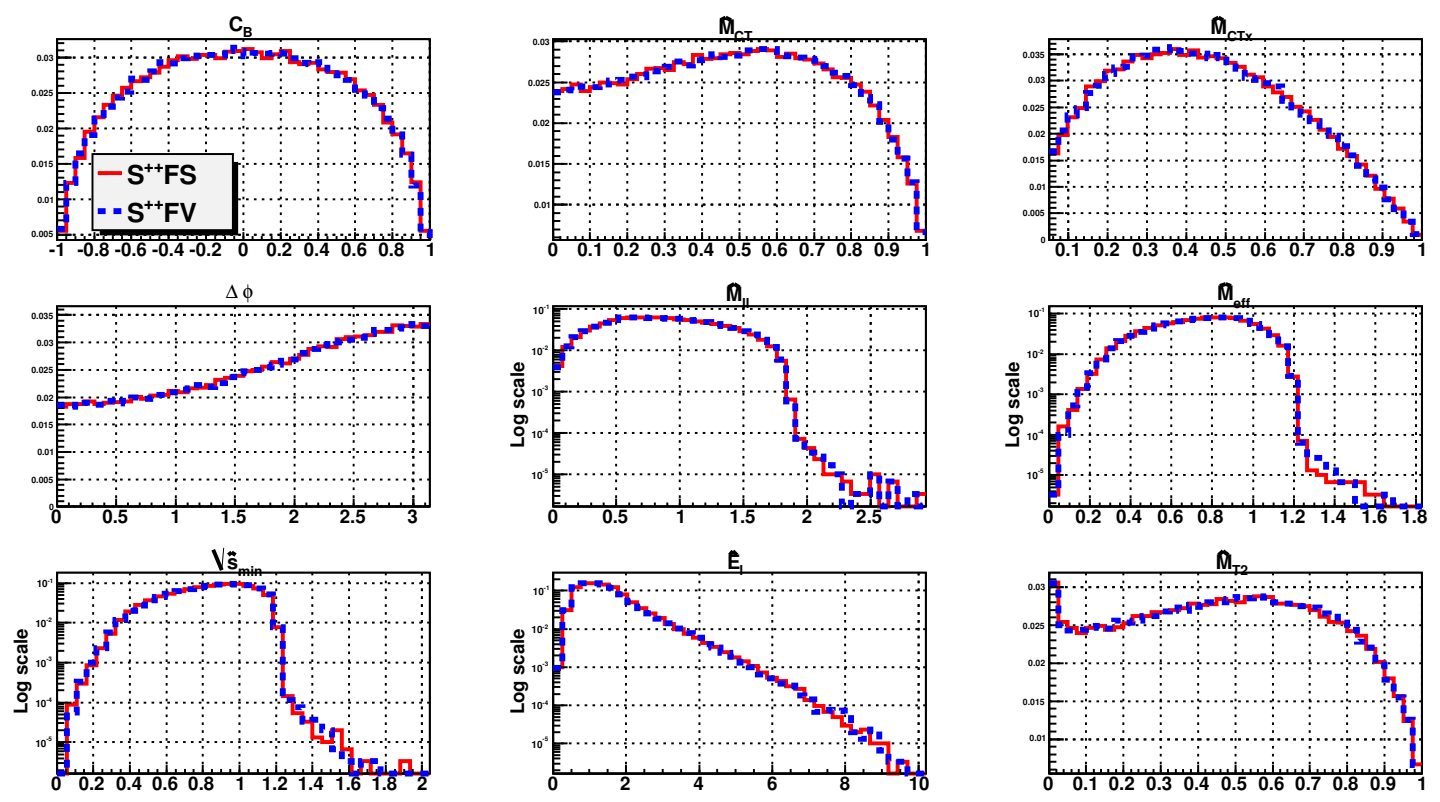

Figure 11. The same as figure 10, but for the SFS and SFV spin scenarios with a scalar particle $A$. Here the threshold behavior for $\mathcal{C}_{\mathrm{B}}$ is obtained with $y \sim 0$, so unlike figure 10 , here we choose $M_{C}=5 \mathrm{GeV}$.
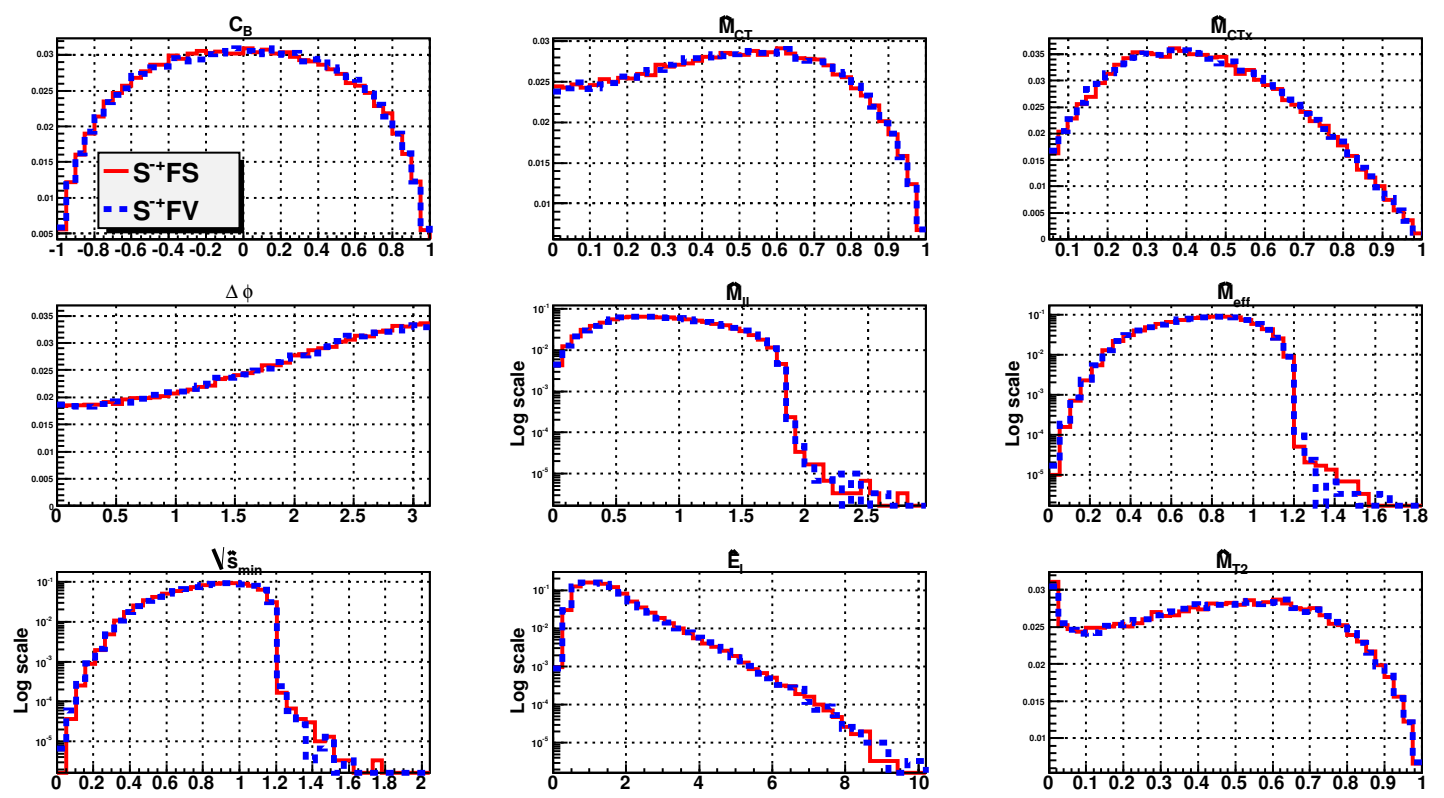

Figure 12. The same as figure 11, but for a pseudoscalar particle $A$.

In conclusion of this section, we can summarize its main two lessons as follows:

- For the spin scenarios where particle $B$ is a fermion, the two situations in which it will be quite difficult to determine the spin of particle $C$ are: 



Figure 13. The same as figure 10, but for the VSF and VVF spin scenarios with $y \sim 1\left(M_{C}=\right.$ $495 \mathrm{GeV})$.
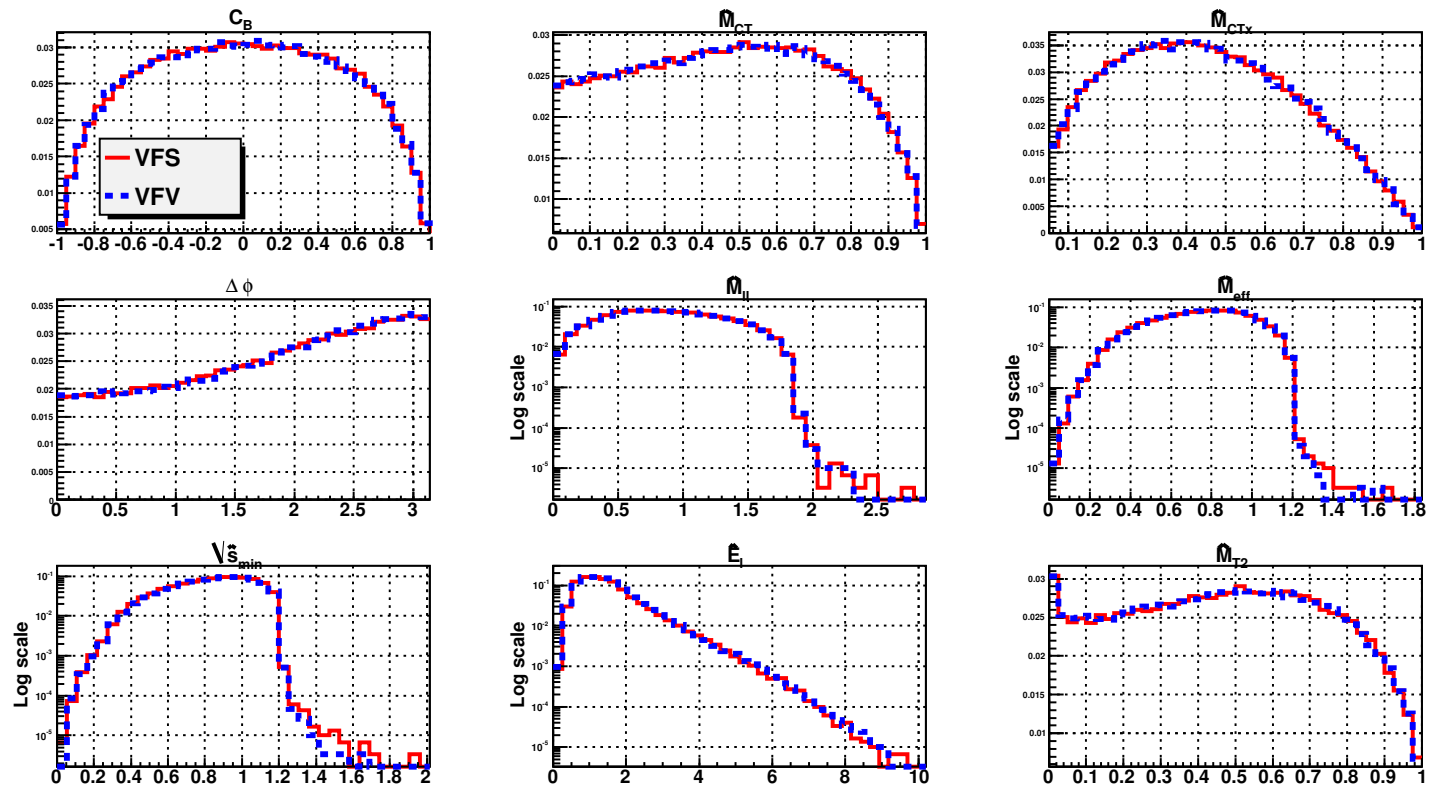

Figure 14. The same as figure 10, but for the VFS and VFV spin scenarios with $\alpha=\beta=\gamma=0$ and $y \sim 0\left(M_{C}=5 \mathrm{GeV}\right)$.

1. Vectorlike couplings between $B$ and $C$ with $\gamma=0$. The presence of chiral couplings is a necessary condition to observe spin correlations [12, 18, 20, 27].

2. Models with $y \sim 0$, where particle $C$ is highly boosted. When $C$ is a vector boson, it is longitudinally polarized and effectively behaves as a scalar. 
- For the spin scenarios where particle $B$ is a boson, it may be difficult to determine whether that boson is a spin 0 or spin 1 particle. Models with $\gamma=0$ and/or $y \sim 1$ are again the most problematic.

\section{Discussion}

In this paper we investigated the antler event topology of figure 1 with regards to spin effects in the kinematic distributions of the two visible particles. Given the simplicity of figure 1, one might have hoped to be able to describe analytically the observable kinematic distributions, including explicitly the effects of spin correlations. Unfortunately, this is not the case, and spin studies must be done numerically by template methods. Nevertheless, useful physics intuition can be developed in the two $\sqrt{s}$ extremes, namely, at threshold $\sqrt{s} \sim 2 M_{B}$ and at $\sqrt{s} \rightarrow \infty$. In section 6 we analyzed the asymptotic analytical expressions for the $\mathcal{C}_{\mathrm{B}}$ distribution, which helped us identify in section 7 the difficult cases for spin discrimination. In table 4 we identified pairs of spin configurations which can easily mimic each other, and the exact circumstances when this is most likely to occur. Typically, the chirality $\gamma$ of the $B C \ell$ coupling and the mass splitting between $B$ and $C$ play an important role - the spin correlations tend to be diminished when $\gamma=0$ and depending on case,

- For the spin scenarios where particle $B$ is a fermion: $M_{C} \sim M_{B}$.

- For the spin scenarios where particle $B$ is a boson: $M_{C} \ll M_{B}$.

While our discussion centered mostly on the $\mathcal{C}_{\mathrm{B}}$ variable, which has been most often utilized for spin studies, we also considered 8 other kinematic variables, which were previously developed mainly in the context of mass determination. Using the popular examples of SUSY and MUED, we investigated their power for spin discrimination. We generally found that some variables are better suited for spin studies than others. Among the better performers were $\mathcal{C}_{\mathrm{B}}, \Delta \varphi, M_{\ell^{+} \ell^{-}}, M_{\text {eff }}$ and $\sqrt{s}_{\text {min }}$. It is interesting to note the complementarity between the $\mathcal{C}_{\mathrm{B}}$ variable and the others in this group. As shown in figure 6 , the $\mathcal{C}_{\mathrm{B}}$ distribution becomes most sensitive to spins at high energies, where the correlation (5.10) is manifest. In contrast, the distributions of the other variables are probing the different $\sqrt{s}$ behavior near threshold (see, e.g. figure 3). Therefore it appears that a multifaceted approach, utilizing a number of different and complementary variables, would be most beneficial for spin determinations.

\section{Acknowledgments}

We thank A. Barr for insightful comments. LE has been supported by the German Ministry of Education and Research (BMBF) under contract no. 05H09WWE and 05H09PAE, and thanks the theory group at the University of Florida for hospitality during the completion of this project. The work of KM is supported by a US Department of Energy grant DEFG02-97ER41029. MP is supported by a CERN-Korea fellowship. 


\section{A Derivation of the $\mathcal{C}_{\mathrm{B}}$ angular distribution at threshold}

In this appendix, we show the derivation of the $\mathcal{C}_{\mathrm{B}}$ angular distributions in the threshold limit $\sqrt{s} \rightarrow 2 M_{B}$. According to (5.11), in that case the $\mathcal{C}_{\mathrm{B}}$ variable can be expressed in terms of the pseudorapidities of the visible particles in the corresponding CMB1 and CMB2 frames as

$$
\mathcal{C}_{\mathrm{B}}=\tanh \left(\frac{\eta_{1}^{\prime}-\eta_{2}^{\prime}}{2}\right)
$$

with $\eta_{1}^{\prime}$ and $\eta_{2}^{\prime}$ defined in (2.8). Since the initial state at the LHC is symmetric (both beams are proton beams), one should symmetrize the $\mathcal{C}_{\mathrm{B}}$ distribution with respect to $\mathcal{C}_{\mathrm{B}} \leftrightarrow-\mathcal{C}_{\mathrm{B}}$, since we do not know which proton beam the initial state quark came from.

\section{A.1 $\mathcal{C}_{\mathrm{B}}$ distribution with no spin correlation}

In the pure phase space limit,

$$
\begin{aligned}
\frac{\mathrm{d} N}{\mathrm{~d} \mathcal{C}_{\mathrm{B}}} & \propto \iiint \mathrm{d} \Omega^{*} \mathrm{~d} \Omega_{1}^{\prime} \mathrm{d} \Omega_{2}^{\prime}|\mathcal{M}|^{2} \delta\left(\mathcal{C}_{\mathrm{B}}-\mathcal{C}_{\mathrm{B}}\left[\Theta^{*}, \varphi^{*}, \eta^{*}, \phi_{i}^{\prime}, \eta_{i}^{\prime}\right]\right) \\
& \propto \iint_{-\infty}^{\infty} \mathrm{d} \eta_{1}^{\prime} \mathrm{d} \eta_{2}^{\prime} \operatorname{sech}^{2} \eta_{1}^{\prime} \operatorname{sech}^{2} \eta_{2}^{\prime} \delta\left(\mathcal{C}_{\mathrm{B}}-\tanh \left(\frac{\eta_{1}^{\prime}-\eta_{2}^{\prime}}{2}\right)\right) \\
& \propto \int_{-\infty}^{\infty} \mathrm{d} \eta_{2}^{\prime}\left\{\cosh \left(\eta_{1}^{\prime(0)}-\eta_{2}^{\prime}\right)+1\right\} \operatorname{sech}^{2} \eta_{1}^{\prime(0)} \operatorname{sech}^{2} \eta_{2}^{\prime},
\end{aligned}
$$

where $\eta_{1}^{\prime(0)}$ is the zero of the argument of the delta function

$$
\mathcal{C}_{\mathrm{B}}-\tanh \left(\frac{\eta_{1}^{\prime}-\eta_{2}^{\prime}}{2}\right)=0 \Longleftrightarrow \eta_{1}^{\prime(0)}=\eta_{2}^{\prime}+\ln \left[\frac{1+\mathcal{C}_{\mathrm{B}}}{1-\mathcal{C}_{\mathrm{B}}}\right]
$$

After integrating over $\eta_{2}^{\prime}$, we get the following unit-normalized distribution

$$
\mathrm{J}_{\mathrm{PS}}=\frac{\mathrm{d} N}{\mathrm{~d} \mathcal{C}_{\mathrm{B}}}=\frac{1-\mathcal{C}_{\mathrm{B}}^{2}}{4 \mathcal{C}_{\mathrm{B}}^{3}}\left\{-2 \mathcal{C}_{\mathrm{B}}+\left(1+\mathcal{C}_{\mathrm{B}}^{2}\right) \ln \left[\frac{1+\mathcal{C}_{\mathrm{B}}}{1-\mathcal{C}_{\mathrm{B}}}\right]\right\}
$$

which is eq. (5.12). This result is already symmetrized with respect to $\mathcal{C}_{\mathrm{B}} \leftrightarrow-\mathcal{C}_{\mathrm{B}}$.

\section{A.2 $\mathcal{C}_{\mathrm{B}}$ distribution when particle $B$ is a fermion}

When particle $B$ is a fermion, the matrix element has the generic form

$$
|\mathcal{M}|^{2} \propto 1+A\left(\cos \theta_{1}^{\prime}-\cos \theta_{2}^{\prime}\right)+\left(B_{1} \cos \Delta \phi^{\prime}+B_{2} \sin \Delta \phi^{\prime}\right) \sin \theta_{1}^{\prime} \sin \theta_{2}^{\prime}+C \cos \theta_{1}^{\prime} \cos \theta_{2}^{\prime} .
$$

The $B_{1}$ and $B_{2}$ terms integrate out to zero and we get

$$
\begin{aligned}
\frac{\mathrm{d} N}{\mathrm{~d} \mathcal{C}_{\mathrm{B}}} & \propto \iiint \mathrm{d} \Omega^{*} \mathrm{~d} \Omega_{1}^{\prime} \mathrm{d} \Omega_{2}^{\prime}|\mathcal{M}|^{2} \delta\left(\mathcal{C}_{\mathrm{B}}-\mathcal{C}_{\mathrm{B}}\left[\Theta^{*}, \varphi^{*}, \eta^{*}, \phi_{i}^{\prime}, \eta_{i}^{\prime}\right]\right) \\
& =\frac{1}{N_{\text {tot }}}\left(\frac{\mathrm{d} N_{\mathrm{PS}}}{\mathrm{d} \mathcal{C}_{\mathrm{B}}}+A \frac{\mathrm{d} N_{A}}{\mathrm{~d} \mathcal{C}_{\mathrm{B}}}+C \frac{\mathrm{d} N_{C}}{\mathrm{~d} \mathcal{C}_{\mathrm{B}}}\right),
\end{aligned}
$$

where

$$
\frac{\mathrm{d} N_{\mathrm{PS}}}{\mathrm{d} \mathcal{C}_{\mathrm{B}}} \propto \iint \mathrm{d} \Omega_{1}^{\prime} \mathrm{d} \Omega_{2}^{\prime} \delta\left(\mathcal{C}_{\mathrm{B}}-\mathcal{C}_{\mathrm{B}}\left[\eta_{1}^{\prime}, \eta_{2}^{\prime}\right]\right)
$$


is the pure phase space contribution already derived in section A.2, while the remaining two contributions are

$$
\begin{aligned}
& \frac{\mathrm{d} N_{A}}{\mathrm{~d} \mathcal{C}_{\mathrm{B}}} \propto \iint \mathrm{d} \Omega_{1}^{\prime} \mathrm{d} \Omega_{2}^{\prime}\left(\cos \theta_{1}^{\prime}-\cos \theta_{2}^{\prime}\right) \delta\left(\mathcal{C}_{\mathrm{B}}-\mathcal{C}_{\mathrm{B}}\left[\eta_{1}^{\prime}, \eta_{2}^{\prime}\right]\right), \\
& \frac{\mathrm{d} N_{C}}{\mathrm{~d} \mathcal{C}_{\mathrm{B}}} \propto \iint \mathrm{d} \Omega_{1}^{\prime} \mathrm{d} \Omega_{2}^{\prime} \cos \theta_{1}^{\prime} \cos \theta_{2}^{\prime} \delta\left(\mathcal{C}_{\mathrm{B}}-\mathcal{C}_{\mathrm{B}}\left[\eta_{1}^{\prime}, \eta_{2}^{\prime}\right]\right)
\end{aligned}
$$

The contribution from (A.8) vanishes after the symmetrization $\mathcal{C}_{\mathrm{B}} \leftrightarrow-\mathcal{C}_{\mathrm{B}}$ and we are left to evaluate

$$
\begin{aligned}
\frac{\mathrm{d} N_{C}}{\mathrm{~d} \mathcal{C}_{\mathrm{B}}} & \propto \iint \mathrm{d} \Omega_{1}^{\prime} \mathrm{d} \Omega_{2}^{\prime} \cos \theta_{1}^{\prime} \cos \theta_{2}^{\prime} \delta\left(\mathcal{C}_{\mathrm{B}}-\mathcal{C}_{\mathrm{B}}\left[\eta_{1}^{\prime}, \eta_{2}^{\prime}\right]\right) \\
& \propto \iint_{-\infty}^{\infty} \mathrm{d} \eta_{1}^{\prime} \mathrm{d} \eta_{2}^{\prime} \operatorname{sech}^{2} \eta_{1}^{\prime} \operatorname{sech}^{2} \eta_{2}^{\prime} \tanh \eta_{1}^{\prime} \tanh \eta_{2}^{\prime} \delta\left(\mathcal{C}_{\mathrm{B}}-\tanh \left(\frac{\eta_{1}^{\prime}-\eta_{2}^{\prime}}{2}\right)\right) \\
& =\int_{-\infty}^{\infty} \mathrm{d} \eta_{2}^{\prime}\left\{\cosh \left(\eta_{1}^{\prime(0)}-\eta_{2}^{\prime}\right)+1\right\} \operatorname{sech}^{2} \eta_{1}^{(0)} \tanh \eta_{1}^{\prime(0)} \operatorname{sech}^{2} \eta_{2}^{\prime} \tanh \eta_{2}^{\prime} \\
& =\frac{2}{1-\mathcal{C}_{\mathrm{B}}^{2}} \int_{-\infty}^{\infty} \mathrm{d} \eta_{2}^{\prime}\left\{\frac{\operatorname{sech}^{2} \eta_{2}^{\prime} \tanh \eta_{2}^{\prime} \tanh \left(\eta_{2}^{\prime}+\ln \left[\frac{1+\mathcal{C}_{\mathrm{B}}}{1-\mathcal{C}_{\mathrm{B}}}\right]\right)}{\cosh ^{2}\left(\eta_{2}^{\prime}+\ln \left[\frac{1+\mathcal{C}_{\mathrm{B}}}{1-\mathcal{C}_{\mathrm{B}}}\right]\right)}\right\}
\end{aligned}
$$

Upon integrating over $\eta_{2}^{\prime}$, this gives the contribution which is due to spin correlations. we use a subscript $\mathrm{F}$ for it as a reminder that it corresponds to the case of a fermion particle $B$ :

$$
\frac{\mathrm{d} N}{\mathrm{~d} \mathcal{C}_{\mathrm{B}}}=\frac{1}{N_{\text {tot }}}\left(\frac{\mathrm{d} N_{\mathrm{PS}}}{\mathrm{d} \mathcal{C}_{\mathrm{B}}}+C \frac{\mathrm{d} N_{\mathrm{F}}}{\mathrm{d} \mathcal{C}_{\mathrm{B}}}\right)=\mathrm{J}_{\mathrm{PS}}+\frac{C}{4} \mathrm{~J}_{\mathrm{F}}
$$

where (A.10) evaluates to

$$
\mathrm{J}_{\mathrm{F}}=\frac{\mathrm{d} N_{\mathrm{F}}}{\mathrm{d} \mathcal{C}_{\mathrm{B}}}=\frac{1-\mathcal{C}_{\mathrm{B}}^{2}}{4 \mathcal{C}_{\mathrm{B}}^{5}}\left\{6 \mathcal{C}_{\mathrm{B}}+4 \mathcal{C}_{\mathrm{B}}^{3}+6 \mathcal{C}_{\mathrm{B}}^{5}-\left(3+\mathcal{C}_{\mathrm{B}}^{2}+\mathcal{C}_{\mathrm{B}}^{4}+3 \mathcal{C}_{\mathrm{B}}^{6}\right) \ln \left(\frac{1+\mathcal{C}_{\mathrm{B}}}{1-\mathcal{C}_{\mathrm{B}}}\right)\right\},
$$

which is the result in (6.11). The prefactor $C$ in (A.11) depends on the particular spin configuration, as seen in eqs. (6.2), (6.3), (6.6), (6.7).

\section{A.3 $\mathcal{C}_{\mathrm{B}}$ distribution in the SVF scenario}

In the SVF spin scenario, the matrix element has the form

$$
\begin{aligned}
|\mathcal{M}|^{2} \propto & 1+\frac{(1-y)^{2}}{1+2 y}\left(\cos \Delta \phi^{\prime} \sin \theta_{1}^{\prime} \sin \theta_{2}^{\prime}-\cos \theta_{1}^{\prime} \cos \theta_{2}^{\prime}\right)^{2} \\
& +\frac{2 \gamma^{2}}{1+2 y}\left(\cos \Delta \phi^{\prime} \sin \theta_{1}^{\prime} \sin \theta_{2}^{\prime}-\cos \theta_{1}^{\prime} \cos \theta_{2}^{\prime}\right) .
\end{aligned}
$$

The $\mathcal{C}_{\mathrm{B}}$ distribution is

$$
\begin{aligned}
\frac{\mathrm{d} N}{\mathrm{~d} \mathcal{C}_{\mathrm{B}}} & \propto \iiint \mathrm{d} \Omega^{*} \mathrm{~d} \Omega_{1}^{\prime} \mathrm{d} \Omega_{2}^{\prime}|\mathcal{M}|^{2} \delta\left(\mathcal{C}_{\mathrm{B}}-\mathcal{C}_{\mathrm{B}}\left[\Theta^{*}, \varphi^{*}, \eta^{*}, \phi_{i}^{\prime}, \eta_{i}^{\prime}\right]\right) \\
& =\frac{1}{N_{\text {tot }}}\left(\frac{\mathrm{d} N_{\mathrm{PS}}}{\mathrm{d} \mathcal{C}_{\mathrm{B}}}+\frac{(1-y)^{2}}{1+2 y} \frac{\mathrm{d} N_{\mathrm{V}}}{\mathrm{d} \mathcal{C}_{\mathrm{B}}}-\frac{2 \gamma^{2}}{1+2 y} \frac{\mathrm{d} N_{\mathrm{F}}}{\mathrm{d} \mathcal{C}_{\mathrm{B}}}\right)
\end{aligned}
$$


where $\frac{\mathrm{d} N_{\mathrm{PS}}}{\mathrm{d} \mathcal{C}_{\mathrm{B}}}$ is the pure phase space contribution (A.4), while $\frac{\mathrm{d} N_{\mathrm{F}}}{\mathrm{d} \mathcal{C}_{\mathrm{B}}}$ is the contribution (A.12) from the helicity structure of the $B \ell C$ interaction. The new term here $\frac{d N_{\mathrm{V}}}{\mathrm{d} \mathcal{C}_{\mathrm{B}}}$ is given by

$$
\frac{\mathrm{d} N_{\mathrm{V}}}{\mathrm{d} \mathcal{C}_{\mathrm{B}}} \propto \iint \mathrm{d} \Omega_{1}^{\prime} \mathrm{d} \Omega_{2}^{\prime}\left(\cos \Delta \phi^{\prime} \sin \theta_{1}^{\prime} \sin \theta_{2}^{\prime}-\cos \theta_{1}^{\prime} \cos \theta_{2}^{\prime}\right)^{2} \delta\left(\mathcal{C}_{\mathrm{B}}-\mathcal{C}_{\mathrm{B}}\left[\eta_{1}^{\prime}, \eta_{2}^{\prime}\right]\right)
$$

After integrating out $\phi_{i}^{\prime}$, we get

$$
\begin{aligned}
\frac{\mathrm{d} N_{\mathrm{V}}}{\mathrm{d} \mathcal{C}_{\mathrm{B}}} & \propto \iint \mathrm{d} \cos \theta_{1}^{\prime} \mathrm{d} \cos \theta_{2}^{\prime}\left(\frac{1}{2} \sin ^{2} \theta_{1}^{\prime} \sin ^{2} \theta_{2}^{\prime}+\cos ^{2} \theta_{1}^{\prime} \cos ^{2} \theta_{2}^{\prime}\right) \delta\left(\mathcal{C}_{\mathrm{B}}-\mathcal{C}_{\mathrm{B}}\left[\eta_{1}^{\prime}, \eta_{2}^{\prime}\right]\right) \\
& \propto \iint_{-\infty}^{\infty} \mathrm{d} \eta_{1}^{\prime} \mathrm{d} \eta_{2}^{\prime} \operatorname{sech}^{4} \eta_{1}^{\prime} \operatorname{sech}^{4} \eta_{2}^{\prime}\left(\frac{1}{2}+\sinh ^{2} \eta_{1}^{\prime} \sinh ^{2} \eta_{2}^{\prime}\right) \delta\left(\mathcal{C}_{\mathrm{B}}-\tanh \left(\frac{\eta_{1}^{\prime}-\eta_{2}^{\prime}}{2}\right)\right) \\
& =\int_{-\infty}^{\infty} \mathrm{d} \eta_{2}^{\prime}\left\{\cosh \left(\eta_{1}^{\prime(0)}-\eta_{2}^{\prime}\right)+1\right\} \operatorname{sech}^{4} \eta_{1}^{(0)} \operatorname{sech}^{4} \eta_{2}^{\prime}\left(\frac{1}{2}+\sinh ^{2} \eta_{1}^{\prime(0)} \sinh ^{2} \eta_{2}^{\prime}\right) \\
& =\frac{2}{1-\mathcal{C}_{\mathrm{B}}^{2}} \int_{-\infty}^{\infty} \mathrm{d} \eta_{2}^{\prime}\left\{\frac{\operatorname{sech}^{4} \eta_{2}^{\prime}\left(\frac{1}{2}+\sinh ^{2} \eta_{2}^{\prime} \sinh ^{2}\left(\eta_{2}^{\prime}+\ln \left[\frac{1+\mathcal{C}_{\mathrm{B}}}{1-\mathcal{C}_{\mathrm{B}}}\right]\right)\right)}{\cosh ^{4}\left(\eta_{2}^{\prime}+\ln \left[\frac{1+\mathcal{C}_{\mathrm{B}}}{1-\mathcal{C}_{\mathrm{B}}}\right]\right)}\right\} .
\end{aligned}
$$

Finally, after integrating over $\eta_{2}^{\prime}$, we get (6.4)

$$
\begin{aligned}
\frac{\mathrm{d} N}{\mathrm{~d} \mathcal{C}_{\mathrm{B}}} & =\frac{1}{N_{\mathrm{tot}}}\left(\frac{\mathrm{d} N_{\mathrm{PS}}}{\mathrm{d} \mathcal{C}_{\mathrm{B}}}+\frac{(1-y)^{2}}{1+2 y} \frac{\mathrm{d} N_{\mathrm{V}}}{\mathrm{d} \mathcal{C}_{\mathrm{B}}}-\frac{2 \gamma^{2}}{1+2 y} \frac{\mathrm{d} N_{\mathrm{F}}}{\mathrm{d} \mathcal{C}_{\mathrm{B}}}\right) \\
& =\frac{3(1+2 y)}{(2+y)^{2}}\left(\mathrm{~J}_{\mathrm{PS}}+\frac{(1-y)^{2}}{4(1+2 y)} \mathrm{J}_{\mathrm{V}}-\frac{\gamma^{2}}{2(1+2 y)} \mathrm{J}_{\mathrm{F}}\right),
\end{aligned}
$$

where the new contribution is given by $(6.12)$

$$
\begin{aligned}
\mathrm{J}_{\mathrm{V}}=\frac{\mathrm{d} N_{V}}{\mathrm{~d} \mathcal{C}_{\mathrm{B}}}= & \frac{1-\mathcal{C}_{\mathrm{B}}^{2}}{48 \mathcal{C}_{\mathrm{B}}^{7}} \cdot\left\{-2 \mathcal{C}_{\mathrm{B}}\left(45+22 \mathcal{C}_{\mathrm{B}}^{4}+45 \mathcal{C}_{\mathrm{B}}^{8}\right)\right. \\
& \left.+3\left(15-5 \mathcal{C}_{\mathrm{B}}^{2}+6 \mathcal{C}_{\mathrm{B}}^{4}+6 \mathcal{C}_{\mathrm{B}}^{6}-5 \mathcal{C}_{\mathrm{B}}^{8}+15 \mathcal{C}_{\mathrm{B}}^{10}\right) \ln \left(\frac{1+\mathcal{C}_{\mathrm{B}}}{1-\mathcal{C}_{\mathrm{B}}}\right)\right\} .
\end{aligned}
$$

\section{A.4 $\mathcal{C}_{\mathrm{B}}$ distribution in the VVF scenario}

After integrating out $\phi_{i}^{\prime}$ from the matrix element, we get

$$
\begin{aligned}
\iint \mathrm{d} \phi_{1}^{\prime} \mathrm{d} \phi_{2}^{\prime}|\mathcal{M}|^{2} \propto & 1-\frac{(1-y)^{2}}{1+y} \cos ^{2} \theta_{1}^{\prime} \cos ^{2} \theta_{2}^{\prime}-\frac{y(1-y)}{2(1+y)}\left(\cos ^{2} \theta_{1}^{\prime}+\cos ^{2} \theta_{2}^{\prime}\right) \\
& +\frac{\alpha \gamma}{1+y}\left\{1-(1-y) \cos \theta_{1}^{\prime} \cos \theta_{2}^{\prime}\right\}\left(\cos \theta_{1}^{\prime}+\cos \theta_{2}^{\prime}\right) .
\end{aligned}
$$

The $\mathcal{C}_{\mathrm{B}}$ distribution is given by

$$
\begin{aligned}
\frac{\mathrm{d} N}{\mathrm{~d} \mathcal{C}_{\mathrm{B}}} & \propto \iiint \mathrm{d} \Omega^{*} \mathrm{~d} \Omega_{1}^{\prime} \mathrm{d} \Omega_{2}^{\prime}|\mathcal{M}|^{2} \delta\left(\mathcal{C}_{\mathrm{B}}-\mathcal{C}_{\mathrm{B}}\left[\Theta^{*}, \varphi^{*}, \eta^{*}, \phi_{i}^{\prime}, \eta_{i}^{\prime}\right]\right) \\
& =\frac{1}{N_{\text {tot }}}\left\{\frac{\mathrm{d} N_{\mathrm{PS}}}{\mathrm{d} \mathcal{C}_{\mathrm{B}}}-\frac{(1-y)^{2}}{1+y} \frac{\mathrm{d} N_{\mathrm{V} 1}}{\mathrm{~d} \mathcal{C}_{\mathrm{B}}}-\frac{y(1-y)}{2(1+y)} \frac{\mathrm{d} N_{\mathrm{V} 2}}{\mathrm{~d} \mathcal{C}_{\mathrm{B}}}+\frac{\alpha \gamma}{1+y} \frac{\mathrm{d} N_{\mathrm{CV}}}{\mathrm{d} \mathcal{C}_{\mathrm{B}}}\right\}
\end{aligned}
$$


but the last term integrates out to zero:

$$
\begin{aligned}
\frac{\mathrm{d} N_{\mathrm{CV}}}{\mathrm{d} \mathcal{C}_{\mathrm{B}}} \propto & \iint \mathrm{d} \cos \theta_{1}^{\prime} \mathrm{d} \cos \theta_{2}^{\prime}\left(\cos \theta_{1}^{\prime}+\cos \theta_{2}^{\prime}\right)\left\{1-(1-y) \cos \theta_{1}^{\prime} \cos \theta_{2}^{\prime}\right\} \delta\left(\mathcal{C}_{\mathrm{B}}-\mathcal{C}_{\mathrm{B}}\left[\eta_{1}^{\prime}, \eta_{2}^{\prime}\right]\right) \\
\propto & \iint_{-\infty}^{\infty} \mathrm{d} \eta_{1}^{\prime} \mathrm{d} \eta_{2}^{\prime} \operatorname{sech}^{3} \eta_{1}^{\prime} \operatorname{sech}^{3} \eta_{2}^{\prime} \sinh \left(\eta_{1}^{\prime}+\eta_{2}^{\prime}\right)\left\{1-(1-y) \tanh \eta_{1}^{\prime} \tanh \eta_{2}^{\prime}\right\} \times \\
& \times \delta\left(\mathcal{C}_{\mathrm{B}}-\tanh \left(\frac{\eta_{1}^{\prime}-\eta_{2}^{\prime}}{2}\right)\right) \\
= & 0 .
\end{aligned}
$$

Thus, in the VVF case, the chiralities $\alpha$ and $\gamma$ do not have an effect on the $\mathcal{C}_{\mathrm{B}}$ distribution at threshold. The second term in (A.20) is

$$
\begin{aligned}
\frac{\mathrm{d} N_{\mathrm{V} 1}}{\mathrm{~d} \mathcal{C}_{\mathrm{B}}} & \propto \iint \mathrm{d} \cos \theta_{1}^{\prime} \mathrm{d} \cos \theta_{2}^{\prime} \cos ^{2} \theta_{1}^{\prime} \cos ^{2} \theta_{2}^{\prime} \delta\left(\mathcal{C}_{\mathrm{B}}-\mathcal{C}_{\mathrm{B}}\left[\eta_{1}^{\prime}, \eta_{2}^{\prime}\right]\right) \\
& \propto \iint_{-\infty}^{\infty} \mathrm{d} \eta_{1}^{\prime} \mathrm{d} \eta_{2}^{\prime} \operatorname{sech}^{2} \eta_{1}^{\prime} \operatorname{sech}^{2} \eta_{2}^{\prime} \tanh ^{2} \eta_{1}^{\prime} \tanh ^{2} \eta_{2}^{\prime} \delta\left(\mathcal{C}_{\mathrm{B}}-\tanh \left(\frac{\eta_{1}^{\prime}-\eta_{2}^{\prime}}{2}\right)\right) \\
& =\int_{-\infty}^{\infty} \mathrm{d} \eta_{2}^{\prime}\left\{\cosh \left(\eta_{1}^{\prime(0)}-\eta_{2}^{\prime}\right)+1\right\} \operatorname{sech}^{2} \eta_{1}^{\prime(0)} \operatorname{sech}^{2} \eta_{2}^{\prime} \tanh ^{2} \eta_{1}^{\prime(0)} \tanh ^{2} \eta_{2}^{\prime} \\
& =\frac{2}{1-\mathcal{C}_{\mathrm{B}}} \int_{-\infty}^{\infty} \mathrm{d} \eta_{2}^{\prime}\left\{\frac{\operatorname{sech}^{2} \eta_{2}^{\prime} \tanh ^{2} \eta_{2}^{\prime} \tanh ^{2}\left(\eta_{2}^{\prime}+\ln \left[\frac{1+\mathcal{C}_{\mathrm{B}}}{1-\mathcal{C}_{\mathrm{B}}}\right]\right)}{\cosh ^{2}\left(\eta_{2}^{\prime}+\ln \left[\frac{1+\mathcal{C}_{\mathrm{B}}}{1-\mathcal{C}_{\mathrm{B}}}\right]\right)}\right\}
\end{aligned}
$$

After integrating out $\eta_{2}^{\prime}$, we get (6.13)

$$
\begin{aligned}
\mathrm{J}_{\mathrm{V} 1}=\frac{\mathrm{d} N_{\mathrm{V} 1}}{\mathrm{~d} \mathcal{C}_{\mathrm{B}}}= & \frac{1-\mathcal{C}_{\mathrm{B}}^{2}}{24 \mathcal{C}_{\mathrm{B}}^{7}} \cdot\left\{-2 \mathcal{C}_{\mathrm{B}}\left(15+8 \mathcal{C}_{\mathrm{B}}^{2}+10 \mathcal{C}_{\mathrm{B}}^{4}+8 \mathcal{C}_{\mathrm{B}}^{6}+15 \mathcal{C}_{\mathrm{B}}^{8}\right)\right. \\
& \left.+3\left(5+\mathcal{C}_{\mathrm{B}}^{2}+2 \mathcal{C}_{\mathrm{B}}^{4}+2 \mathcal{C}_{\mathrm{B}}^{6}+\mathcal{C}_{\mathrm{B}}^{8}+5 \mathcal{C}_{\mathrm{B}}^{10}\right) \ln \left(\frac{1+\mathcal{C}_{\mathrm{B}}}{1-\mathcal{C}_{\mathrm{B}}}\right)\right\}
\end{aligned}
$$

By a similar calculation, we obtain (6.14)

$$
\mathrm{J}_{\mathrm{V} 2}=\frac{\mathrm{d} N_{\mathrm{V} 2}}{\mathrm{~d} \mathcal{C}_{\mathrm{B}}}=\frac{1}{3 \mathcal{C}_{\mathrm{B}}^{5}}\left\{-2 \mathcal{C}_{\mathrm{B}}\left(3+\mathcal{C}_{\mathrm{B}}^{2}-\mathcal{C}_{\mathrm{B}}^{4}-3 \mathcal{C}_{\mathrm{B}}^{6}\right)+3\left(1-\mathcal{C}_{\mathrm{B}}^{8}\right) \ln \left(\frac{1+\mathcal{C}_{\mathrm{B}}}{1-\mathcal{C}_{\mathrm{B}}}\right)\right\}
$$

In total, we get the answer in (6.8)

$$
\begin{aligned}
\frac{\mathrm{d} N}{\mathrm{~d} \mathcal{C}_{\mathrm{B}}} & =\frac{1}{N_{\mathrm{tot}}}\left\{\frac{\mathrm{d} N_{\mathrm{PS}}}{\mathrm{d} \mathcal{C}_{\mathrm{B}}}-\frac{(1-y)^{2}}{1+y} \frac{\mathrm{d} N_{\mathrm{V} 1}}{\mathrm{~d} \mathcal{C}_{\mathrm{B}}}-\frac{y(1-y)}{2(1+y)} \frac{\mathrm{d} N_{\mathrm{V} 2}}{\mathrm{~d} \mathcal{C}_{\mathrm{B}}}\right\} \\
& =\frac{9(1+y)}{2(2+y)^{2}}\left\{\mathrm{~J}_{\mathrm{PS}}-\frac{(1-y)^{2}}{4(1+y)} \mathrm{J}_{\mathrm{V} 1}-\frac{y(1-y)}{8(1+y)} \mathrm{J}_{\mathrm{V} 2}\right\}
\end{aligned}
$$

\section{A.5 $\mathcal{C}_{\mathrm{B}}$ distribution in SUSY and MUED}

For SUSY (MSSM), the threshold behavior of $\mathcal{C}_{\mathrm{B}}$ is given by $(6.10)$, since $B$ is a scalar particle (slepton):

$$
\frac{\mathrm{d} N}{\mathrm{~d} \mathcal{C}_{\mathrm{B}}}=\mathrm{J}_{\mathrm{PS}} .
$$


For the minimal UED model (MUED), the matrix element is

$$
|\mathcal{M}|^{2} \propto 1-A_{1}\left(\cos \theta_{1}^{\prime}-\cos \theta_{2}^{\prime}\right)-A_{2} \cos \theta_{1}^{\prime} \cos \theta_{2}^{\prime},
$$

with

$$
\begin{aligned}
& A_{1}=2\left(\frac{1-2 y}{1+2 y}\right) \frac{\left(3-\frac{M_{Z}^{2}}{M_{B}^{2}} C_{W}^{2}\right)}{1+\left(3-\frac{M_{Z}^{2}}{M_{B}^{2}} C_{W}^{2}\right)^{2}}, \\
& A_{2}=\left(\frac{1-2 y}{1+2 y}\right)^{2} .
\end{aligned}
$$

$M_{Z}$ is the mass of the $Z$ boson and $C_{W} \equiv \cos \theta_{W}$ is the cosine of the Weinberg angle $\theta_{W}$. Comparing (A.27) to (A.5), we find $C=-A_{2}$ and from (A.11) we get

$$
\frac{\mathrm{d} N}{\mathrm{~d} \mathcal{C}_{\mathrm{B}}}=\mathrm{J}_{\mathrm{PS}}-\frac{1}{4}\left(\frac{1-2 y}{1+2 y}\right)^{2} \mathrm{~J}_{\mathrm{F}} .
$$

Open Access. This article is distributed under the terms of the Creative Commons Attribution License which permits any use, distribution and reproduction in any medium, provided the original author(s) and source are credited.

\section{References}

[1] A.J. Barr and C.G. Lester, A review of the mass measurement techniques proposed for the large hadron collider, J. Phys. G 37 (2010) 123001 [arXiv:1004.2732] [INSPIRE].

[2] A. Barr, T. Khoo, P. Konar, K. Kong, C. Lester, et al., Guide to transverse projections and mass-constraining variables, Phys. Rev. D 84 (2011) 095031 [arXiv:1105.2977] [InSPIRE].

[3] A. Barr, Determining the spin of supersymmetric particles at the LHC using lepton charge asymmetry, Phys. Lett. B 596 (2004) 205 [hep-ph/0405052] [INSPIRE].

[4] M. Battaglia, A. Datta, A. De Roeck, K. Kong and K.T. Matchev, Contrasting supersymmetry and universal extra dimensions at the CLIC multi-TeV $e^{+} e^{-}$collider, JHEP 07 (2005) 033 [hep-ph/0502041] [INSPIRE].

[5] J.M. Smillie and B.R. Webber, Distinguishing spins in supersymmetric and universal extra dimension models at the large hadron collider, JHEP 10 (2005) 069 [hep-ph/0507170] [INSPIRE].

[6] A. Datta, K. Kong and K.T. Matchev, Discrimination of supersymmetry and universal extra dimensions at hadron colliders, Phys. Rev. D 72 (2005) 096006 [Erratum ibid. D 72 (2005) 119901] [hep-ph/0509246] [INSPIRE].

[7] A. Datta, G.L. Kane and M. Toharia, Is it SUSY?, hep-ph/0510204 [InSPIRE].

[8] A. Barr, Measuring slepton spin at the LHC, JHEP 02 (2006) 042 [hep-ph/0511115] [INSPIRE].

[9] P. Meade and M. Reece, Top partners at the LHC: spin and mass measurement, Phys. Rev. D 74 (2006) 015010 [hep-ph/0601124] [INSPIRE]. 
[10] A. Alves, O. Eboli and T. Plehn, It's a gluino, Phys. Rev. D 74 (2006) 095010 [hep-ph/0605067] [INSPIRE].

[11] C. Athanasiou, C.G. Lester, J.M. Smillie and B.R. Webber, Distinguishing spins in decay chains at the large hadron collider, JHEP 08 (2006) 055 [hep-ph/0605286] [INSPIRE].

[12] L.-T. Wang and I. Yavin, Spin measurements in cascade decays at the LHC, JHEP 04 (2007) 032 [hep-ph/0605296] [INSPIRE].

[13] J.M. Smillie, Spin correlations in decay chains involving $W$ bosons, Eur. Phys. J. C 51 (2007) 933 [hep-ph/0609296] [INSPIRE].

[14] C. Kilic, L.-T. Wang and I. Yavin, On the existence of angular correlations in decays with heavy matter partners, JHEP 05 (2007) 052 [hep-ph/0703085] [INSPIRE].

[15] A. Alves and O. Eboli, Unravelling the sbottom spin at the CERN LHC, Phys. Rev. D 75 (2007) 115013 [arXiv: 0704.0254] [INSPIRE].

[16] C. Csáki, J. Heinonen and M. Perelstein, Testing gluino spin with three-body decays, JHEP 10 (2007) 107 [arXiv:0707.0014] [INSPIRE].

[17] M.R. Buckley, H. Murayama, W. Klemm and V. Rentala, Discriminating spin through quantum interference, Phys. Rev. D 78 (2008) 014028 [arXiv:0711.0364] [INSPIRE].

[18] L.-T. Wang and I. Yavin, A review of spin determination at the LHC, Int. J. Mod. Phys. A 23 (2008) 4647 [arXiv:0802.2726] [INSPIRE].

[19] M.R. Buckley, B. Heinemann, W. Klemm and H. Murayama, Quantum interference effects among helicities at LEP-II and Tevatron, Phys. Rev. D 77 (2008) 113017 [arXiv:0804.0476] [INSPIRE].

[20] M. Burns, K. Kong, K.T. Matchev and M. Park, A general method for model-independent measurements of particle spins, couplings and mixing angles in cascade decays with missing energy at hadron colliders, JHEP 10 (2008) 081 [arXiv:0808.2472] [INSPIRE].

[21] W.S. Cho, K. Choi, Y.G. Kim and C.B. Park, $M_{T 2}$-assisted on-shell reconstruction of missing momenta and its application to spin measurement at the LHC, Phys. Rev. D 79 (2009) 031701 [arXiv:0810.4853] [INSPIRE].

[22] O. Gedalia, S.J. Lee and G. Perez, Spin determination via third generation cascade decays, Phys. Rev. D 80 (2009) 035012 [arXiv:0901.4438] [INSPIRE].

[23] F. Boudjema and R.K. Singh, A model independent spin analysis of fundamental particles using azimuthal asymmetries, JHEP 07 (2009) 028 [arXiv:0903.4705] [INSPIRE].

[24] W. Ehrenfeld, A. Freitas, A. Landwehr and D. Wyler, Distinguishing spins in decay chains with photons at the large hadron collider, JHEP 07 (2009) 056 [arXiv:0904.1293] [INSPIRE].

[25] L. Edelhauser, W. Porod and R.K. Singh, Spin discrimination in three-body decays, JHEP 08 (2010) 053 [arXiv: 1005.3720] [INSPIRE].

[26] D. Horton, Reconstructing events with missing transverse momentum at the LHC and its application to spin measurement, arXiv:1006.0148 [INSPIRE].

[27] H.-C. Cheng, Z. Han, I.-W. Kim and L.-T. Wang, Missing momentum reconstruction and spin measurements at hadron colliders, JHEP 11 (2010) 122 [arXiv:1008.0405] [INSPIRE].

[28] M.R. Buckley and M.J. Ramsey-Musolf, Diagnosing spin at the LHC via vector boson fusion, JHEP 09 (2011) 094 [arXiv: 1008.5151] [INSPIRE]. 
[29] C.-Y. Chen and A. Freitas, General analysis of signals with two leptons and missing energy at the large hadron collider, JHEP 02 (2011) 002 [arXiv:1011.5276] [INSPIRE].

[30] M.M. Nojiri and J. Shu, Two jets and missing $E_{T}$ signature to determine the spins of the new particles, JHEP 06 (2011) 047 [arXiv:1101.2701] [INSPIRE].

[31] G. Moortgat-Pick, K. Rolbiecki and J. Tattersall, Early spin determination at the LHC?, Phys. Lett. B 699 (2011) 158 [arXiv:1102.0293] [INSPIRE].

[32] L. Edelhauser, W. Porod and R.K. Singh, Effects of anomalous couplings on spin determination in cascade decays, arXiv:1109.3369 [INSPIRE].

[33] T. Melia, Spin before mass at the LHC, JHEP 01 (2012) 143 [arXiv:1110.6185] [INSPIRE].

[34] C.-Y. Chen and A. Freitas, General analysis of decay chains with three-body decays involving missing energy, JHEP 01 (2012) 124 [arXiv:1110.6192] [INSPIRE].

[35] M. Burns, K. Kong, K.T. Matchev and M. Park, Using subsystem $M_{T 2}$ for complete mass determinations in decay chains with missing energy at hadron colliders, JHEP 03 (2009) 143 [arXiv:0810.5576] [INSPIRE].

[36] T. Han, I.-W. Kim and J. Song, Kinematic cusps: determining the missing particle mass at colliders, Phys. Lett. B 693 (2010) 575 [arXiv:0906.5009] [InSPIRE].

[37] J. Alwall, A. Freitas and O. Mattelaer, Measuring sparticles with the matrix element, AIP Conf. Proc. 1200 (2010) 442 [arXiv:0910.2522] [INSPIRE].

[38] J. Alwall, A. Freitas and O. Mattelaer, The matrix element method and QCD radiation, Phys. Rev. D 83 (2011) 074010 [arXiv: 1010.2263] [INSPIRE].

[39] B. Gripaios, Transverse observables and mass determination at hadron colliders, JHEP 02 (2008) 053 [arXiv:0709.2740] [INSPIRE].

[40] A.J. Barr, B. Gripaios and C.G. Lester, Weighing WIMPs with kinks at colliders: invisible particle mass measurements from endpoints, JHEP 02 (2008) 014 [arXiv:0711.4008] [INSPIRE].

[41] A.J. Barr, B. Gripaios and C.G. Lester, Transverse masses and kinematic constraints: from the boundary to the crease, JHEP 11 (2009) 096 [arXiv:0908.3779] [INSPIRE].

[42] K.T. Matchev, F. Moortgat, L. Pape and M. Park, Precision sparticle spectroscopy in the inclusive same-sign dilepton channel at LHC, Phys. Rev. D 82 (2010) 077701 [arXiv: 0909.4300] [INSPIRE].

[43] K.T. Matchev and M. Park, A general method for determining the masses of semi-invisibly decaying particles at hadron colliders, Phys. Rev. Lett. 107 (2011) 061801 [arXiv:0910.1584] [INSPIRE].

[44] P. Konar, K. Kong, K.T. Matchev and M. Park, Superpartner mass measurement technique using $1 D$ orthogonal decompositions of the Cambridge transverse mass variable $M_{T 2}$, Phys. Rev. Lett. 105 (2010) 051802 [arXiv:0910.3679] [INSPIRE].

[45] P. Konar, K. Kong, K.T. Matchev and M. Park, Dark matter particle spectroscopy at the LHC: generalizing $M_{T 2}$ to asymmetric event topologies, JHEP 04 (2010) 086 [arXiv: 0911.4126] [INSPIRE].

[46] T. Cohen, E. Kuflik and K.M. Zurek, Extracting the dark matter mass from single stage cascade decays at the LHC, JHEP 11 (2010) 008 [arXiv:1003.2204] [INSPIRE]. 
[47] W.S. Cho, W. Klemm and M.M. Nojiri, Mass measurement in boosted decay systems at hadron colliders, Phys. Rev. D 84 (2011) 035018 [arXiv:1008.0391] [INSPIRE].

[48] C.B. Park, Reconstructing the heavy resonance at hadron colliders, Phys. Rev. D 84 (2011) 096001 [arXiv:1106.6087] [InSPIRE].

[49] H.-C. Cheng and J. Gu, Measuring invisible particle masses using a single short decay chain, JHEP 10 (2011) 094 [arXiv: 1109.3471] [INSPIRE].

[50] S. Chang, K.Y. Lee and J. Song, The second Kaluza-Klein neutral Higgs bosons in the minimal universal extra dimension model, Phys. Lett. B 707 (2012) 144 [arXiv:1101.0670] [INSPIRE].

[51] M. Baumgart, T. Hartman, C. Kilic and L.-T. Wang, Discovery and measurement of sleptons, binos and winos with a $Z^{\prime}, J H E P 11$ (2007) 084 [hep-ph/0608172] [INSPIRE].

[52] O. Eboli, C.S. Fong, J. Gonzalez-Fraile and M. Gonzalez-Garcia, Determination of the spin of new resonances in electroweak gauge boson pair production at the $\mathrm{LHC}$, Phys. Rev. D 83 (2011) 095014 [arXiv: 1102.3429] [INSPIRE].

[53] G. Burdman, B.A. Dobrescu and E. Ponton, Resonances from two universal extra dimensions, Phys. Rev. D 74 (2006) 075008 [hep-ph/0601186] [INSPIRE].

[54] B.A. Dobrescu, K. Kong and R. Mahbubani, Leptons and photons at the LHC: cascades through spinless adjoints, JHEP 07 (2007) 006 [hep-ph/0703231] [INSPIRE].

[55] H.-C. Cheng, K.T. Matchev and M. Schmaltz, Bosonic supersymmetry? getting fooled at the CERN LHC, Phys. Rev. D 66 (2002) 056006 [hep-ph/0205314] [INSPIRE].

[56] CMS collaboration, G. Bayatian et al., CMS technical design report, volume II: physics performance, J. Phys. G 34 (2007) 995 [INSPIRE].

[57] D.R. Tovey, On measuring the masses of pair-produced semi-invisibly decaying particles at hadron colliders, JHEP 04 (2008) 034 [arXiv:0802.2879] [INSPIRE].

[58] D. Tovey, Measuring the SUSY mass scale at the LHC, Phys. Lett. B 498 (2001) 1 [hep-ph/0006276] [INSPIRE].

[59] P. Konar, K. Kong and K.T. Matchev, $\sqrt{\hat{s}}_{\text {min }}$ : a global inclusive variable for determining the mass scale of new physics in events with missing energy at hadron colliders, JHEP 03 (2009) 085 [arXiv: 0812.1042] [InSPIRE].

[60] P. Konar, K. Kong, K.T. Matchev and M. Park, RECO level $\sqrt{s}_{\min }$ and subsystem $\sqrt{s}_{\min }$ : improved global inclusive variables for measuring the new physics mass scale in $/ E_{T}$ events at hadron colliders, JHEP 06 (2011) 041 [arXiv: 1006. 0653] [INSPIRE].

[61] C. Lester and D. Summers, Measuring masses of semiinvisibly decaying particles pair produced at hadron colliders, Phys. Lett. B 463 (1999) 99 [hep-ph/9906349] [INSPIRE].

[62] W.S. Cho, K. Choi, Y.G. Kim and C.B. Park, Measuring superparticle masses at hadron collider using the transverse mass kink, JHEP 02 (2008) 035 [arXiv:0711.4526] [INSPIRE].

[63] W.S. Cho, J.E. Kim and J.-H. Kim, Amplification of endpoint structure for new particle mass measurement at the LHC, Phys. Rev. D 81 (2010) 095010 [arXiv:0912.2354] [INSPIRE].

[64] M.E. Cabrera, J.A. Casas and J.A. Casas, Understanding and improving the effective mass for LHC searches, arXiv:1207.0435 [INSPIRE]. 
[65] S. Kawabata, Y. Shimizu, Y. Sumino and H. Yokoya, Boost-invariant leptonic observables and reconstruction of parent particle mass, Phys. Lett. B 710 (2012) 658 [arXiv:1107.4460] [INSPIRE].

[66] C. Rogan, Kinematical variables towards new dynamics at the LHC, arXiv:1006.2727 [INSPIRE].

[67] J. Alwall, P. Demin, S. de Visscher, R. Frederix, M. Herquet, et al., MadGraph/MadEvent v4: the new web generation, JHEP 09 (2007) 028 [arXiv:0706.2334] [INSPIRE]. 